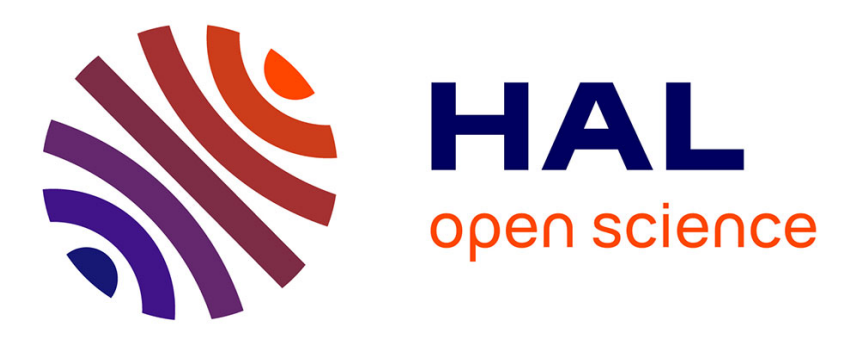

\title{
Numerical study of cavitating flows with thermodynamic effect
}

\author{
Eric Goncalvès da Silva, Regiane. Fortes Patella
}

\section{To cite this version:}

Eric Goncalvès da Silva, Regiane . Fortes Patella. Numerical study of cavitating flows with thermodynamic effect. Computers and Fluids, 2010, 39 (1), pp.99-113. 10.1016/j.compfluid.2009.07.009 . hal-00530261

\section{HAL Id: hal-00530261 \\ https://hal.science/hal-00530261}

Submitted on 29 Oct 2010

HAL is a multi-disciplinary open access archive for the deposit and dissemination of scientific research documents, whether they are published or not. The documents may come from teaching and research institutions in France or abroad, or from public or private research centers.
L'archive ouverte pluridisciplinaire HAL, est destinée au dépôt et à la diffusion de documents scientifiques de niveau recherche, publiés ou non, émanant des établissements d'enseignement et de recherche français ou étrangers, des laboratoires publics ou privés. 


\title{
Numerical study of cavitating flows with thermodynamic effect
}

\author{
Eric Goncalvès *, Regiane Fortes Patella \\ LEGI Grenoble-INP, 1025 rue de la Piscine, 38400 St Martin d'Heres, France
}

\begin{abstract}
Thermodynamic effects play an important role in the cavitation dynamics of cryogenic fluids. Such flows are characterized by strong variations in fluid properties with the temperature. A compressible, multiphase, one-fluid formulation that accounts for the energy balance and variable thermodynamics properties of the fluid is described. A preconditioning method for low Mach number areas is presented. Cavitation phenomenon is modelled by two different liquid-vapour mixture equations of state (EOS). Mathematical and thermodynamic properties are studied. Numerical results are given for a Venturi geometry with freon R-114 fluid and comparisons are made with experimental data.
\end{abstract}

Key words: Cavitation, Homogeneous Model, Thermodynamic Effect, Equation of State, RANS Simulations

\section{Introduction}

The simulation and the prediction of cavitation in cryogenic fluids is of critical importance for the efficient design and performance of turbopumps in

* Corresponding author.

Email address: Eric.Goncalves@grenoble-inp.fr (Eric Goncalvès ). 
rocket propulsion systems. With thermosensible fluids, thermal effects and strong variations in fluid properties are observed, which alter the cavity characteristics. Sheet cavitation involves complex interactions between the vapour and liquid phases. The cavity is sustained by a continuous vaporization and condensation processes whose rates are determined by the geometry and flow conditions. The shape and size of the cavity are dependent on the physical properties of the working fluid and the local fluid pressure, velocity and temperature.

For cryogenic fluids, the liquid-vapour density ratio is lower than that of typical fluids (cold water) and consequently more liquid mass has to vaporize to sustain a cavity. Therefore evaporative cooling effects are more pronounced and the temperature of the liquid in the immediate vicinity of the liquidvapour interface is depressed below the free-stream temperature. Because of the strong variation of thermodynamic properties (vapour pressure, density), the temperature depression, negligible in water, is quite substantial. The local cooling effect delays the cavitation phenomenon and reduces the local vapour pressure of the fluid, which leads to a lower observed cavity pressure. Typical temperature depressions in cryogenic fluids are only one to two Kelvin, this corresponds to changes in vapour pressure of a factor of two.

First studies about thermal effects were generally focussed on obtaining correlations for temperature depression as a function of flow conditions and liquid properties. Classical methods include the $B$-factor theory $[17,27,36]$ to characterize the sensitivity of fluids to thermodynamic effects, and the entrainment theory $[5,16]$. The $B$-factor method is based on the ratio of vapour volume to liquid volume affected by the vaporization process. A simple heat balance between the two phases can estimate the scale of temperature difference $\Delta T^{*}$ caused by thermal effects. The $B$-factor is estimated as the ratio between the 
actual temperature drop and $\Delta T^{*}$ :

$$
\Delta T^{*}=\frac{\rho_{V} L_{v a p}}{\rho_{L} C_{p_{L}}} \quad \text { and } \quad B=\frac{\Delta T}{\Delta T^{*}}
$$

where $L_{v a p}$ is the latent heat, $\rho_{L}$ and $\rho_{V}$ densities for the liquid and vapour phases, respectively. $C_{p_{L}}$ represents the specific heat. Values of the temperature difference $\Delta T^{*}$ are given in Table 1 for water and freon R-114.

Various numerical models have been developed to investigate thermodynamic effects in cavitating flows and more generally two-phase flows with phase transition in metastable liquids. A liquid initially in thermodynamic equilibrium can reach a metastable state where the temperature is higher than the saturated one (superheated liquid) or where the pressure is lower than the saturated one (stretched liquid). Different classes of models are available in the literature within the framework of the continuum modelling method. This method makes no attempt to track the liquid and vapour interface but treats the flow as two-phases with an averaged mixture density, which continuously varies between the liquid and vapour extremes. These two-phase models are becoming more and more popular because of their abilities to include the physics of cavitating flows. In its implementation, there are different approaches according to the assumptions made: equilibrium models versus non-equilibrium models, the one-fluid method or homogeneous models versus the two-fluid method.

The two-fluid approach assumes that both phases co-exist at every point in the flow field and each phase is governed by its own set of conservation laws. For example, a seven-equation model has been used for inviscid high speed cavitating applications and two-phase Riemann problems [22,33]. Because the exchanges of mass, momentum and energy are treated explicitly as transfer terms, these models can take into account the physical details occurring in the cavitation phenomenon such as mass exchange, thermal transfer and sur- 
face tension. However, the transfer terms have to be known; such quantities are usually very difficult to obtain. Moreover, these models can involve nonconservative terms that is a numerical problem.

The one-fluid method treats the cavitating flows as a mixture of two fluids behaving as one. The governing equations are composed by three conservation laws written for the mixture. These models are based on the assumption of local kinematic equilibrium between phases (the local velocity is the same for both phases) and local thermodynamic equilibrium between the two components (local temperature, pressure and free energy equality between phases), which constitute the homogeneous equilibrium models (HEM). Moreover, vaporization or condensation processes are assumed to be instantaneous. An equation of state (EOS) is necessary to define the thermodynamic behaviour of the mixture. Different closure relations that link the pressure to the temperature and the density have been proposed, from tabular EOS (for ebullition applications [7]), barotropic EOS (for cavitation in liquid hydrogen [8] or in hot water [29]), to more sophisticated EOS (for cavitation in octane [10] or in refrigerant R-114 [31]). Small non-equilibrium effects (spinodal values in the metastable area) can be introduced in the EOS compared to an isothermal thermodynamic path. When non-equilibrium effect becomes important, additional equations are needed for an accurate prediction.

Hybrid or reduced models are intermediate models between one-fluid and twofluid ones, based on four or five equations. A five-equation model can be obtained from a simplification of a seven-equation two-fluid model. It is composed by four conservation laws (two mass balance, one mixture momentum, one mixture energy) plus a non-conservative equation for the void ratio. It involves two temperature and then is able to capture temperature non-equilibrium effects. Such models have been used for inviscid high speed cavitating applications and cavitation pocket in fuel injector nozzles [34]. A four-equation model has been used for ebullition applications and flashing flows: the homogeneous relaxation model (HRM). It is composed by three conservation laws 
for the mixture augmented by a void ratio equation with a relaxation term. The source term involves a relaxation time that is the time for the system to regain its thermodynamic equilibrium state. This time is very difficult to determine and is estimated with experimental data [2-4]. Another formulation of the relaxation term was proposed by [15], based on a constrained convex optimization problem on the mixture entropy. Another four-equation model, very popular to simulate cavitating flows in cold water, has been adapted to cryogenic applications $[1,18,40,42]$. It is obtained by adding a mass equation for the vapour or liquid density including a cavitation source term. The main difficulty is related to the formulation of the source term and the tunable parameters involved for the vaporization and condensation processes (different sets of parameters are presented in [40]).

The present paper proposes a simple homogeneous formulation with two different mixture EOS: an EOS derived from a barotropic law and a model based on the stiffened gas law. For both EOS, mathematical properties such as hyperbolicity and convexity are studied for the inviscid Euler equations. Numerical simulations are performed with an implicit preconditioned compressible RANS solver. The boundary conditions are treated with preconditioned characteristic relations. We test different transport-equation turbulence models associated with the Boussinesq assumption and calibrated for single-phase flows. The models and numerical techniques are assessed by comparing the numerical results with experimental data on two Venturi geometries. The running fluid is freon R-114 $\left(\mathrm{C}_{2} \mathrm{Cl}_{2} F_{4}\right)$. Local analyses with void ratio profiles and wall temperature depression are proposed.

In the following, we first describe the essential elements of the governing equations, the modelling concepts, the numerical schemes, and then present the results. 


\section{Governing equations and models}

The numerical simulations were carried out using an in-house CFD code solving the one-fluid compressible RANS system. First, we present the one-fluid formulation for Euler equations, with the associated preconditioned method and studies of mathematical and thermodynamic properties. Second, the complete RANS system is presented.

\subsection{The one-fluid Euler equations}

The homogeneous model assumes strong coupling of the phases and moving at the same velocity components. The phases are assumed in close proximity to each other so that heat transfer would occur instantaneously maintaining the phases in thermal equilibrium. Furthermore, the disperse phase behaves quasi-statically to change in pressure and the mixture are assumed in constant pressure. Therefore the phases share the same temperature $T$ and the same pressure $P$.

The evolution of the two-phase flow can be described by Euler equations that employ the representative flow properties as unknowns just as a single-phase problem. The mixture density $\rho$ is defined by:

$$
\rho=\alpha \rho_{V}+(1-\alpha) \rho_{L}
$$

where $\rho_{L}$ and $\rho_{V}$ are respectively the liquid and vapour densities. The void ratio $\alpha$ characterizes the volume of vapour in each cell: $\alpha=1$ means that the cell is completely filled by vapour; inversely, a complete liquid cell is represented by $\alpha=0$. Liquid and vapour phases are characterized by their thermodynamic properties. On each cell, the unknowns are calculated by averaging them by the volume occupied. 
In order to simplify the formulation, we present below the one-dimensional equations, expressed in conservative variables $w=(\rho, \rho u, \rho E)$ :

$$
\begin{aligned}
\frac{\partial \rho}{\partial t}+\frac{\partial \rho u}{\partial x} & =0 \\
\frac{\partial(\rho u)}{\partial t}+\frac{\partial\left(\rho u^{2}+P\right)}{\partial x} & =0 \\
\frac{\partial(\rho E)}{\partial t}+\frac{\partial(\rho u H)}{\partial x} & =0
\end{aligned}
$$

where $E=e+u^{2} / 2$ denotes the total energy and $H=h+u^{2} / 2$ the total enthalpy; $e$ the internal energy, $h$ the enthalpy, which are related to the void ratio and the liquid and vapour values:

$$
\begin{aligned}
& \rho e=\alpha \rho_{V} e_{V}+(1-\alpha) \rho_{L} e_{L} \\
& \rho h=\alpha \rho_{V} h_{V}+(1-\alpha) \rho_{L} h_{L}
\end{aligned}
$$

To close the system, an equation of state (EOS) and a thermal relation are necessary to link the pressure and the temperature to the internal energy and the density. The difficulty with this homogeneous approach is to specify an EOS that covers all possible fluid states: pure liquid, two-phase mixture and pure gas. Different EOS can be used in pure phases and in the mixture. In the present study, we propose a stiffened gas EOS for the pure phases and two formulations for the two-phase mixture: a mixture of stiffened gas and a modified barotropic EOS.

Moreover, mathematical and thermodynamic properties should be analyzed: the hyperbolicity of the inviscid system has to be verified (i.e. the sound of speed $c^{2}>0$ ) and a suitable mixture EOS has to be convex to respect the second principle of thermodynamics, and to obtain thermodynamically stable states. Given a two-phase mixture with an entropy, a thermodynamic state is said to be stable if it is an absolute maximum of the entropy and it is metastable if it is a relative maximum of the entropy. This induces the fact that the entropy $s$ of a given state must always ensure the following criterion: 
$d^{2} s<0$ (condition of concave entropy, the Hessian matrix of $s$ has to be definite negative). Moreover, the concavity of $s$ is equivalent to the convexity of $e$ (i.e. the Hessian matrix of internal energy has to be positive definite).

\subsubsection{The pure phases EOS}

\section{Model description}

For pure phases, we use the convex stiffened gas $(\mathrm{SG})$ equation of state (see $[21]):$

$$
\begin{aligned}
P(\rho, e) & =(\gamma-1) \rho(e-q)-\gamma P_{\infty} \\
P(\rho, T) & =\rho(\gamma-1) C_{v} T-P_{\infty} \\
T(\rho, h) & =\frac{h-q}{C_{p}}
\end{aligned}
$$

where $\gamma=C_{p} / C_{v}$ is the heat capacity ratio, $C_{p}$ and $C_{v}$ are thermal capacities, $q$ the energy of the fluid at a given reference state and $P_{\infty}$ is a constant reference pressure.

The associated speed of sound $c$, defined as $c^{2}=\left(\frac{\partial P}{\partial \rho}\right)_{s}=\frac{\frac{P}{\rho^{2}}-\left(\frac{\partial e}{\partial \rho}\right)_{P}}{\left(\frac{\partial e}{\partial P}\right)_{\rho}}$, can be easily computed:

$$
c^{2}=\gamma \frac{P+P_{\infty}}{\rho}=(\gamma-1) C_{p} T
$$

$c^{2}$ remains strictly positive (for $\gamma>1$ ) that ensures the strict hyperbolicity of the system and the existence of a convex mathematical entropy [14]. The eigenvalues of the system are $u$ and $\lambda_{ \pm}=u \pm c$. 
For low Mach number applications, a well-known problem concerns the stiffness on the solution convergence. In this situation, the dominance of convection terms renders the system stiff and compressible solvers converge slowly. To overcome this difficulty, a preconditioning method is necessary. The physical acoustic waves are replaced by pseudo-acoustic modes that are much closer to the advective velocity, reducing the stiffness and enhancing the convergence. The method is based on the modification of the derivative term by a premultiplication with a suitable preconditioning matrix. With the primitive variables $W=(P, u, e)$ the preconditioned Euler equations can be expressed as:

$$
P_{e}^{-1} \frac{\partial W}{\partial t}+A_{e} \frac{\partial W}{\partial x}=0
$$

We use the preconditioning matrix based on the Turkel approach $[13,39]$ :

$$
P_{e}=\left[\begin{array}{ccc}
\beta^{2} & 0 & 0 \\
0 & 1 & 0 \\
0 & 0 & 1
\end{array}\right] \quad ; \quad A_{e}=\left[\begin{array}{ccc}
u & \rho c^{2} & 0 \\
1 / \rho & u & 0 \\
0 & P / \rho & u
\end{array}\right]
$$

$\beta$ is a parameter on the order of the Mach number. In our study, we have chosen the form given by Choi and Merkle [6]:

$$
\beta^{2}=\min \left[\max \left(M^{2}, K M_{\infty}^{2}\right), 1\right]
$$

This form implies that there is no preconditioning used in transonic and supersonic flow regions (in the mixture). When $\beta^{2}=1$, the preconditioning matrix becomes the identity matrix and the system returns to its classical non preconditioned form. Moreover, for a very small flow velocity, $\beta^{2}$ is not allowed 
to be less than a given percentage of the freestream velocity, determined by the coefficient $K$. For inviscid computations, $K$ is on the order of unity.

The eigenvalues of the preconditioned system are:

$$
u \quad ; \quad \lambda_{ \pm}=\frac{1}{2}\left[u\left(1+\beta^{2}\right) \pm \sqrt{\left(\beta^{2}-1\right)^{2} u^{2}+4 \beta^{2} c^{2}}\right]
$$

For the conservative variables $w=[\rho, \rho u, \rho E]$, the corresponding form is:

$$
P_{c}^{-1} \frac{\partial w}{\partial t}+A_{c} \frac{\partial w}{\partial x}=0
$$

where the preconditioning matrix $P_{c}^{-1}=\frac{\partial w}{\partial W} P_{e}^{-1} \frac{\partial W}{\partial w}$ and $A_{c}$ is the Jacobian matrix of the convective fluxes. Expressions of $R=\frac{\partial w}{\partial W}$ and $R^{-1}=\frac{\partial W}{\partial w}$ are given in appendix A.

The preconditioned matrix can be written as $P_{c}^{-1}=I_{d}+\frac{\left(1-\beta^{2}\right)}{\beta^{2}(e-q)} \times B$ where $I_{d}$ is the identity matrix and the matrix $B$ is:

$$
B=\left[\begin{array}{ccc}
\frac{u^{2}}{2} & -u & 1 \\
\frac{u^{2}}{2} u & -u^{2} & u \\
\frac{u^{2}}{2}(E-q) & -u(E-q) & E-q
\end{array}\right]
$$

The matrix $B$ is idempotent (i.e. $B^{2}=B$ ) and the inverse matrix $P_{c}$ can be easily computed: $P_{c}=I_{d}+\frac{\left(\beta^{2}-1\right)}{(e-q)} \times B$.

In the mixture area, whose models are presented below, the preconditioned matrix is not necessary. Indeed, in the compressible area, $\beta^{2}=1, P_{c}=I_{d}$ and the classical compressible system is integrated. 
Another preconditioner based on the primitive variables $(P, u, S)$ was tested (the formulation is given in [12]), leading to less robust simulations.

\subsubsection{The mixture EOS}

The mixture of stiffened gas EOS - properties

The first cavitation model applied in this study is based on a mixture of stiffened gas. This model was developed and tested for cold water applications [12]. On the basis of the stiffened gas EOS for each pure phase, an expression for the pressure and the temperature can be deduced from the thermal and mechanical equilibrium assumption. These expressions, function of the void ratio $\alpha$, are available in all possible fluid states:

$$
\begin{aligned}
P(\rho, e, \alpha) & =(\gamma(\alpha)-1) \rho(e-q(\alpha))-\gamma(\alpha) P_{\infty}(\alpha) \\
\frac{1}{\gamma(\alpha)-1} & =\frac{\alpha}{\gamma_{V}-1}+\frac{1-\alpha}{\gamma_{L}-1} \\
\rho q(\alpha) & =\alpha \rho_{V} q_{V}+(1-\alpha) \rho_{L} q_{L} \\
P_{\infty}(\alpha) & =\frac{\gamma(\alpha)-1}{\gamma(\alpha)}\left[\alpha \frac{\gamma_{V}}{\gamma_{V}-1} P_{\infty}^{V}+(1-\alpha) \frac{\gamma_{L}}{\gamma_{L}-1} P_{\infty}^{L}\right] \\
T(\rho, h) & =\frac{h_{L}-q_{L}}{C_{p_{L}}}=\frac{h_{V}-q_{V}}{C_{p_{V}}}=\frac{h-q(\alpha)}{C_{p}(\alpha)} \\
\rho C_{p}(\alpha) & =\alpha \rho_{V} C_{p_{V}}+(1-\alpha) \rho_{L} C_{p_{L}}
\end{aligned}
$$

At thermodynamic equilibrium, the two Gibbs free energy $(g=h-T s)$ have to be equal, that implies a relation between $P_{\text {vap }}$ and $T$. The complete formulation is detailed in [34] for a mixture of stiffened gas :

$$
\begin{aligned}
\ln \left(P+P_{\infty}^{L}\right) & =A+\frac{B}{T}+C \ln (T)+D \ln \left(P+P_{\infty}^{V}\right) \\
\frac{d P}{d T}(T) & =\frac{\frac{C}{T}-\frac{B}{T^{2}}}{\frac{1}{P+P_{\infty}^{V}}-\frac{D}{P+P_{\infty}^{L}}}
\end{aligned}
$$

where A, B, C and D are constant. A Newton algorithm can be used to solve the non linear equation. The main characteristic of this model is the pres- 
ence of a "kink", a discontinuity in the derivatives along the saturation curves which separates the single phase and the two-phase domains. Numerically, this full equilibrium model is very stiff and very hard to converge.

To avoid an iterative procedure, we assume that the vapor pressure $P_{v a p}$ varies linearly with the temperature :

$$
P_{v a p}(T)=P_{v a p}\left(T_{r e f}\right)+\frac{d P}{d T}\left(T-T_{r e f}\right)
$$

The constant quantity $\frac{d P}{d T}$ is estimated with a thermodynamic table of freon R-114 (values are given in Table 3). To evaluate the impact of this assumption, we computed the derivative $\frac{d P}{d T}$ of the full equilibrium model (equation (23)), for a temperature $T_{r e f}=293 \mathrm{~K}$. In the mixture of freon, this quantity is almost constant, it varies between 5970 and $6028 \mathrm{~Pa} / \mathrm{K}$. Consequently, the variation of $P_{v a p}$ with the temperature is well quasi-linear.

The void ratio $\alpha$ can be computed with an iterative algorithm from these two relations:

$$
\begin{aligned}
\rho e & =\alpha\left(\rho_{V} e_{V}-\rho_{L} e_{L}\right)+\rho_{L} e_{L} \\
\alpha & =\frac{\rho-\rho_{L}(T)}{\rho_{V}(T)-\rho_{L}(T)}
\end{aligned}
$$

To avoid an iterative procedure, we assume that densities of each phase vary linearly with the temperature :

$$
\begin{aligned}
& \rho_{L}(T)=\rho_{L}^{s a t}\left(T_{r e f}\right)+\frac{d \rho_{L}}{d T}\left(T-T_{r e f}\right) \\
& \rho_{V}(T)=\rho_{V}^{s a t}\left(T_{r e f}\right)+\frac{d \rho_{V}}{d T}\left(T-T_{r e f}\right)
\end{aligned}
$$

The constant quantities $\frac{d \rho_{k}}{d T}$ are evaluated with a thermodynamic table of 
freon R-114 (see Table 2 and Table 3, Section 4.2.1). Equation (26) allows the determination of $\alpha$ with the temperature evaluated at the previous iteration. Thus, the system is closed.

The speed of sound in the mixture can be expressed as a function of the enthalpy of each phase (see appendix B) :

$$
\begin{aligned}
& C_{1}=\frac{1}{\gamma-1}+\frac{\rho_{V} h_{V}-\rho_{L} h_{L}}{\left(\rho_{L}-\rho_{V}\right) \frac{d P}{d T}}\left(\alpha \frac{d \rho_{V}}{d T}+(1-\alpha) \frac{d \rho_{L}}{d T}\right) \\
& \rho c^{2}=\rho\left(\frac{\partial P}{\partial \rho}\right)_{s}=\frac{1}{C_{1}}\left[\frac{\rho_{V} \rho_{L}}{\left(\rho_{L}-\rho_{V}\right)}\left(h_{V}-h_{L}\right)\right]
\end{aligned}
$$

$c^{2}$ is always strictly positive therefore the system is strictly hyperbolic.

The convexity of internal energy is necessary to ensure thermodynamic stability and to respect the entropy growth criterion. The Hessian matrix of $e$ has to be positive definite. With the introduction of these dimensionless parameters:

$$
\lambda=\frac{\rho}{P}\left(\frac{\partial P}{\partial \rho}\right)_{s} \quad ; \quad g=\frac{P}{\rho T^{2}}\left(\frac{\partial T}{\partial s}\right)_{\rho} \quad ; \quad \Gamma=\frac{\rho}{T}\left(\frac{\partial T}{\partial \rho}\right)_{s}
$$

an equivalent condition of convexity was formulated by Perrier [28] for flows with phase transition :

$$
\lambda \geq 0 \quad ; \quad g \geq 0 \quad ; \quad \lambda g-\Gamma^{2} \geq 0
$$

The different parameters can be computed (see Appendix C):

$$
\begin{aligned}
\lambda & =\frac{\rho c^{2}}{P}>0 \\
C_{2} & =\rho C_{p}+\left[\frac{\left(C_{p_{L}}-C_{p_{V}}\right) T+\left(q_{L}-q_{V}\right)}{\rho_{V}-\rho_{L}}\right]\left(\alpha \rho_{L} \frac{d \rho_{V}}{d T}+(1-\alpha) \rho_{V} \frac{d \rho_{L}}{d T}\right) 3 \\
g & =\frac{P\left(1+C_{1}\right)}{T C_{1} C_{2}}
\end{aligned}
$$




$$
\Gamma=\frac{\rho\left(C_{1}+1\right) c^{2}}{T C_{2}}
$$

With parameters presented in Section 4.2.1, the quantities $C_{1}$ and $C_{2}$ are positive therefore $g$ and $\Gamma$ are also positive.

The third condition can be expressed as:

$$
c^{2} \leq \frac{T C_{2}}{\rho C_{1}\left(1+C_{1}\right)}
$$

This condition is also respected for applications considered in the paper.

\section{A modified barotropic EOS - properties}

The second model proposed is based on a sinusoidal barotropic law $[9,12]$. This law is modified by introducing thermal variations. When the pressure becomes smaller than the quantity $P_{v a p}(T)+\Delta P$ and greater than $P_{v a p}(T)-\Delta P$, we have the relation:

$$
P(\alpha, T)=P_{v a p}(T)+\left(\frac{\rho_{L}^{s a t}-\rho_{V}^{s a t}}{2}\right) c_{b a r o}^{2} \operatorname{Arcsin}(A(1-2 \alpha))
$$

where $\Delta P$ represents the pressure width of the law and, for a void ratio value set at 0.5 , the pressure is equal to the vaporization pressure $P_{v a p}(T)$. The constant $A$, close to 1 , allows to avoid infinite value of the speed of sound in pure phases. The quantity $c_{\text {baro }}$ is an adjustable parameter of the model which has the dimension of a velocity. For cold water, $c_{\text {baro }}$ can be interpreted as the minimum speed of sound in the mixture.

As previously with the stiffened gas EOS, we assume that the vaporization pressure varies linearly with the temperature :

$$
P_{v a p}(T)=P_{v a p}\left(T_{r e f}\right)+\frac{d P}{d T}\left(T-T_{r e f}\right)
$$


The void ratio is computed with saturation values of densities:

$$
\alpha=\frac{\rho-\rho_{L}^{s a t}}{\rho_{V}^{s a t}-\rho_{L}^{s a t}}
$$

For an equilibrium two-phase flow, the pressure depends only on the temperature, derivatives of $P$ and $T$ with respect to the density are zero. With this EOS, these quantities are no more equal to zero (their expressions are given in Appendix D), leading to a small thermal effect.

The speed of sound can be expressed as (see Appendix D):

$$
\begin{aligned}
\left(\frac{\partial \rho}{\partial T}\right)_{P} & =-\frac{d P}{d T} \frac{1}{A c_{\text {baro }}^{2}} \cos \left(\frac{P-P_{\text {vap }}(T)}{0.5\left(\rho_{L}^{\text {sat }}-\rho_{V}^{\text {sat }}\right) c_{\text {baro }}^{2}}\right)<0 \\
K_{1} & =\frac{\rho C_{p}}{\frac{d P}{d T}} \\
\left(K_{1}-1\right) c^{2} & =\frac{\rho_{V} \rho_{L}}{\rho\left(\rho_{L}-\rho_{V}\right)}\left(h_{V}-h_{L}\right)-\rho C_{p}\left(\frac{\partial T}{\partial \rho}\right)_{P}
\end{aligned}
$$

With our choice of parameters for the model (values are given in Table 4, Section 4.2 .2$)$, the quantity $K_{1}-1$ is positive and $c^{2}$ is always strictly positive, therefore the system is strictly hyperbolic.

The dimensionless parameters for convexity conditions concerning this approach are (see Appendix E):

$$
\begin{aligned}
\lambda & =\frac{\rho c^{2}}{P}>0 \\
g & =\frac{P}{T\left(\rho C_{p}-\frac{d P}{d T}\right)} \\
\Gamma & =\frac{\rho c^{2}}{T \frac{d P}{d T}}+\frac{\rho}{T}\left(\frac{\partial T}{\partial \rho}\right)_{P}
\end{aligned}
$$

With our choice of parameters, the first two conditions are well respected.

Assuming the cosine value is equal to 1 in the expression of $\left(\frac{\partial T}{\partial \rho}\right)_{P}$ (that is to 
say the pressure $P=P_{v a p}(T)$ ), the third convexity relation can be expressed as:

$$
\begin{array}{r}
K_{2}=c_{\text {baro }}^{2}+\frac{T \frac{d P}{d T}}{2 \rho\left(K_{1}-1\right)} \\
K_{2}+\sqrt{K_{2}^{2}-c_{\text {baro }}^{4}} \leq c^{2} \leq K_{2}+\sqrt{K_{2}^{2}+c_{\text {baro }}^{4}}
\end{array}
$$

This inequality is respected for void ratio values depending on the choice of the parameter $c_{\text {baro }}$. For example, for the freon R-114, if $c_{\text {baro }}=1.48 \mathrm{~m} / \mathrm{s}$, the convexity is verified for void ratio values smaller than 0.8 . The convexity domains are given in Table 4 for different values of the parameter $c_{b a r o}$.

\subsection{Reynolds-Averaged Navier-Stokes equations}

For turbulent computations, the compressible one-fluid RANS equations are used, coupled with a one- or two-equation turbulence model. For low Mach number applications, the inviscid preconditioner presented previously is used (no viscous terms are introduced in the formulation). These equations can be expressed as:

$$
\begin{aligned}
& P_{c}^{-1} \frac{\partial w}{\partial t}+\operatorname{div}\left(F_{c}-F_{v}\right)=S \\
& w=\left(\begin{array}{c}
\rho \\
\rho V \\
\rho E \\
\rho k \\
\rho V \\
\rho \Psi
\end{array}\right) ; F_{c}=\left(\begin{array}{c}
(49) \\
(\rho E+p) V \\
\rho k V \\
\rho \Psi V
\end{array}\right) \quad ; \quad F_{v}=\left(\begin{array}{c}
\left(\overline{\overline{\tau^{v}}}+\overline{\overline{\tau^{t}}}\right) \cdot V-Q^{v}-Q^{t} \\
\overline{\overline{\tau^{v}}}+\overline{\overline{\tau^{t}}} \\
\left(\mu+\mu_{t} / \sigma_{k}\right) \operatorname{grad} k \\
\left(\mu+\mu_{t} / \sigma_{\Psi}\right) \operatorname{grad} \Psi
\end{array}\right)
\end{aligned}
$$


where $w$ denotes the conservative variables, $F_{c}$ and $F_{v}$ the convective and viscous flux densities and $S$ the source terms which concern only the transport equations. $k$ is the turbulent kinetic energy and $\Psi$ is a turbulent variable.

The exact expression of the eddy-viscosity $\mu_{t}$ and the source terms depend on the turbulence model, as well as the constants $\sigma_{k}$ and $\sigma_{\Psi}$.

The total stress tensor $\overline{\bar{\tau}}$ is evaluated following the Stokes hypothesis and the Boussinesq assumption. The total heat flux vector $Q$ is obtained from the Fourier law with the constant Prandtl number hypothesis.

$$
\begin{aligned}
& \left.\overline{\bar{\tau}}=\overline{\overline{\tau^{v}}}+\overline{\overline{\tau^{t}}}=\left(\mu+\mu_{t}\right)\left[\frac{1}{2}\left(\operatorname{grad} V+(\operatorname{grad} V)^{t}\right)-\frac{2}{3}(\operatorname{div} V) \overline{\bar{I}}\right]+\frac{2}{3} k \overline{\bar{I}} 50\right) \\
& Q=Q^{v}+Q^{t}=-\left(\frac{\mu}{P_{r}}+\frac{\mu_{t}}{P_{r t}}\right) C_{p} \operatorname{grad} T
\end{aligned}
$$

In the pure liquid, the viscosity is determined by an exponential law and, in pure vapour, the viscosity follows the Sutherland law. The mixture viscosity is calculated as an arithmetic mean between the liquid and the vapour viscosity:

$$
\begin{aligned}
\mu_{L}(T) & =\mu_{0_{L}} \exp (B / T) \\
\mu_{V}(T) & =\mu_{0_{V}} \sqrt{\frac{T}{293}} \frac{1+T_{S} / 293}{1+T_{S} / T} \\
\mu(T, \alpha) & =\alpha \mu_{V}(T)+(1-\alpha) \mu_{L}(T)
\end{aligned}
$$

where $\mu_{0_{L}}, \mu_{0_{V}}, B$ and $T_{S}$ are constant parameters.

The mixture Prandtl number is also evaluated as an arithmetic mean between the liquid and the vapour value:

$$
P_{r}(\alpha)=\alpha P_{r_{V}}+(1-\alpha) P_{r_{L}}
$$

The turbulent Prandtl number $P_{r t}$ is set at 1 . 


\subsubsection{Turbulence model}

The one-equation Spalart-Allmaras model (SA) [35] was used for this study. Moreover, a comparison with two other models, the Jones-Launder $k-\varepsilon$ model (KE) [20] and the Menter SST $k-\omega$ model (KWSST) [24], is proposed.

\section{Numerical scheme}

The numerical simulations were carried out using an implicit CFD code solving the RANS/turbulent systems for multi-domain structured meshes. This solver is based on a cell-centered finite-volume discretization.

\subsection{Spatial discretization}

The system in integral form is written for a computational cell of volume $\Omega$ limited by a surface $\Sigma$, with an outer normal $n$. It can be expressed as:

$$
P_{c}^{-1} \frac{d}{d t} \int_{\Omega} w d \Omega+\oint_{\Sigma} F_{c} \cdot n d \Sigma-\oint_{\Sigma} F_{v} \cdot n d \Sigma=\int_{\Omega} S d \Omega
$$

Using the finite-volume technique for space discretization, a semi-discrete form of equation (56) can be written as:

$$
P_{c}^{-1} \Omega \frac{\partial w}{\partial t}+\sum_{\text {allfaces }}\left(F_{c}-F_{v}\right) \cdot n \Sigma=\Omega \hat{S}
$$

For the mean flow, the convective flux density vector on a cell face is computed with the Jameson scheme [19] in which the dispersive error is cancelled. The artificial viscosity includes a second-order dissipation term $D_{2}$ and a fourthorder dissipation term $D_{4}$. The use of a preconditioning method modifies these dissipation terms: 


$$
\begin{aligned}
& D_{2}(w)=P_{c}^{-1} \sum_{l=i, j, k} \delta_{l}\left(P_{c} \varepsilon_{l}^{(2)}\left|\lambda_{+}\right| \delta_{l} w\right) \\
& D_{4}(w)=P_{c}^{-1} \sum_{l=i, j, k} \delta_{l}\left(P_{c} \varepsilon_{l}^{(4)}\left|\lambda_{+}\right| \delta_{l}^{3} w\right)
\end{aligned}
$$

where, for each direction $l, \varepsilon_{l}^{(2)}=k^{(2)} \max \left(\nu_{l}, \nu_{l+1}\right)$ and $\varepsilon_{l}^{(4)}=\max \left(0, k^{(4)}-\varepsilon_{l}^{(2)}\right)$, $k^{(2)}$ and $k^{(4)}$ are tunable numerical coefficients. $\lambda_{+}$is the higher eigenvalue of the preconditioned system. $\delta_{l}$ is the difference operator and $\nu_{l}$ represents a sensor based on the pressure gradients. For two-phase flows, $\nu_{l}$ is also based on the density gradients.

The viscous terms are discretized by a second-order space-centered scheme. For the turbulence transport equations, the upwind Roe scheme [30] is used to obtain a more robust method. The second-order accuracy is obtained by introducing a flux-limited dissipation [38].

\subsection{Temporal discretization}

Time integration is achieved using a low-cost implicit method [23]. The implicit method consists in solving a system of equations arising from the linearization of a fully implicit scheme, at each time step. The main feature of this method is that the storage of the Jacobian matrix is completely eliminated, which leads to a low-storage algorithm. More details are given in [12].

The implicit time-integration procedure leads to a system that can be solved directly or iteratively. The direct inversion can be memory intensive and computationally expensive. Therefore, an implicit relaxation procedure is preferred and the point Jacobi relaxation algorithm was chosen.

For the turbulence transport equations, the diffusive flux Jacobian matrix is 
replaced by its spectral radius. The source term needs special treatment [25]. Only the negative part of the source term Jacobian matrix is considered and replaced by its spectral radius. The system obtained is solved with a linealternated Jacobi relaxation algorithm.

\subsection{Inlet and outlet boundary conditions}

The numerical treatment of the boundary conditions is based on the use of the preconditioned characteristic relations. The number of variables to impose at boundaries is given by the number of positive characteristics. The characteristic relations obtained for the preconditioned system, in two-dimensional flows, are:

$$
\begin{aligned}
-c^{2}\left(\rho^{c}-\rho^{s}\right)+\left(P^{c}-P^{s}\right) & =0 \\
V_{t}^{c}-V_{t}^{s} & =0 \\
\left(\lambda_{+}-V_{n}\right)\left(P^{c}-P^{s}\right)+\rho \beta^{2} c^{2}\left(V_{n}^{c}-V_{n}^{s}\right) & =0 \\
\left(\lambda_{-}-V_{n}\right)\left(P^{c}-P^{s}\right)+\rho \beta^{2} c^{2}\left(V_{n}^{c}-V_{n}^{s}\right) & =0
\end{aligned}
$$

The variables with superscript $c$ denote the variables to be computed at the boundary. Variables with superscript $s$ denote the variables obtained by the current numerical scheme. $V_{t}$ and $V_{n}$ are the tangential and the normal component of the mean velocity, respectively.

At inflow, four variables have to be imposed: we chose the stagnation pressure $P_{i}$, the stagnation temperature $T_{i}$, and the direction of the velocity. A Newton algorithm makes it possible to compute the pressure with the characteristic relation (63) and the conservative variables can be evaluated at the boundary. At outflow, only one variable is imposed: the static pressure was chosen. The conservative variables are computed with three characteristic relations (60)(62).

We assumed that inlet and outlet areas are in a pure liquid region. No cavitation appears in these boundaries. 


\subsection{Turbulent variables}

Since the discretization scheme does not ensure the positivity of the turbulent conservative variables, limiters are used to avoid negative values. These limiters are set equal to the corresponding imposed boundary values in the inlet.

\section{Numerical results}

The proposed physical and numerical models were applied in a Venturi geometry, for freon R-114 cavitating flows. All experiments were led at the CREMHyG (Centre d'Essais de Machines Hydrauliques de Grenoble).

\subsection{Experimental conditions}

The R-114 experimental facility of the CREMHyG is a closed loop operating with a reference pressure, obtained by pressurizing a tank with nitrogen gas. The cavitation tunnel was designed to simulate cavitating flows developing on the blades of space turbopump inducers. The loop is fitted with a test section having the shape of a two-dimensional Venturi, characterized by a convergence angle of $4.3^{\circ}$ and a divergence angle of $4^{\circ}$ (Fig. 2). The edge forming the throat of the Venturi is used to fix the separation point of the cavitation cavity. The geometrical data are:

Inlet section: $S_{i}=50 \times 44 \mathrm{~mm}^{2}$ (where the reference pressure is measured)

Throat section: $S_{\text {throat }}=43.7 \times 44 \mathrm{~mm}^{2}$

Length of the test section (chord): $L_{r e f}=252 \mathrm{~mm}$.

This geometry is equipped with three probing holes to take various measure- 
ments. Optical probes and micro-thermocouples are used to evaluate the local void ratio and the wall temperature, respectively. Two different roofs were used: a flat roof and an undulated roof.

The freon R-114 is a thermosensible fluid, which allows to study the thermodynamic effect in ambient conditions. It provides the same temperature depression $\Delta T^{*}$ in comparison with the liquid hydrogen at $T_{r e f}=22 \mathrm{~K}$. The thermodynamic properties at saturation for cold water and freon R-114 are compared in Table 1.

\subsubsection{The flat roof Venturi}

For this geometry, only one inlet velocity was tested. The selected operation point is characterized by the following physical parameters [11,37]:

$V_{\text {inlet }}=14.4 \mathrm{~m} / \mathrm{s}:$ the inlet velocity

$P_{\text {inlet }}=265300 \mathrm{~Pa}:$ the pressure in the inlet section

$T_{\text {ref }}=293 \mathrm{~K}:$ the reference temperature

$\sigma_{\text {inlet }}=\frac{P_{\text {inlet }}-P_{\text {vap }}\left(T_{\text {ref }}\right)}{0.5 \rho V_{\text {inlet }}^{2}} \simeq 0.55:$ the cavitation parameter in the inlet section

$R e_{L_{\text {ref }}}=\frac{V_{\text {inlet }} L_{r e f}}{\nu\left(T_{\text {ref }}\right)}=18.410^{6}:$ the Reynolds number based on $L_{r e f}$ $\Delta T^{*}=1.22 \mathrm{~K}:$ the characteristic temperature depression

With these parameters, a cavity length $L$ around $80 \mathrm{~mm}$ was obtained, with a relatively stable aspect.

\subsubsection{The undulated roof Venturi}

For this geometry, different cavity sheets were studied [26] by varying :

- the inlet velocity $V_{\text {inlet }}$ from 14.4 to $38.2 \mathrm{~m} / \mathrm{s}$

- the reference temperature $T_{\text {ref }}$ from 293 to $303 \mathrm{~K}$

- the $\sigma_{\text {inlet }}$ value from 0.52 to 0.62 


\subsection{Computational parameters and meshes}

\subsubsection{Parameters of the stiffened gas EOS}

The different parameters, evaluated with a R-114 thermodynamic table, are presented in Table 2 and Table 3.

\subsubsection{Parameters of the barotropic EOS}

For pure phases, the stiffened gas EOS is used with the same parameters defined on the previous section. In the mixture area, the different parameters are presented in Table 4 . We tested three $c_{\text {baro }}$ values to study the influence of this parameter. The last column indicates for which void ratio values the convexity conditions are respected.

\subsubsection{The speed of sound in the mixture}

Figure 3 compares the evolution of the speed of sound in the mixture in function of the void ratio for the EOS presented and the Wallis formulation [41]. The Wallis speed of sound is calculated with the assumption of local thermodynamic equilibrium and by neglecting the exchange of mass between the two phases. The formulation is expressed as a weighted harmonic mean of speeds of sound of each phase:

$$
\frac{1}{\rho c^{2}}=\frac{\alpha}{\rho_{V} c_{V}^{2}}+\frac{1-\alpha}{\rho_{L} c_{L}^{2}}
$$

To compare the different speeds, the temperature is assumed to be constant equal to $293 \mathrm{~K}$ and saturation values are used for $\rho_{L}, \rho_{V}, h_{L}$ and $h_{V}$. The speed of sound evaluated "without thermo" means that quantities $\frac{d P}{d T}, \frac{d \rho_{k}}{d T}$ are equal to zero. 
The mixture sound of speed with both EOS is smaller than the Wallis one (for $\alpha=0.5$, there is a factor 4 ), because the mass transfer is accounted. For both EOS, the introduction of thermal variations for the vapour pressure induces a decrease of the speed of sound. Moreover, the speed of sound obtained with both EOS is not symmetric, that is not the case for the standard barotropic law in cold water [12]. The minimum value (around $2.5 \mathrm{~m} / \mathrm{s}$ ) is reached for void ratio close to zero. Finally, for the modified barotropic EOS, the influence of the parameter $c_{\text {baro }}$ is weak for the tested values. This parameter can not be any more interpreted as the minimum of the speed of sound as observed for cold water.

\subsubsection{Meshes}

For both geometries, the grid is a H-type topology. A special contraction is applied in the main flow direction just after the throat to better simulate the two-phase flow area.

For the flat roof Venturi, the mesh contains 193 nodes in the flow direction and 93 in the orthogonal direction (Fig. 4).

For the undulated roof Venturi, the mesh contains 201 nodes in the flow direction and 85 in the orthogonal direction (Fig. 5).

For both mesh, the $y^{+}$values at the center of the first cell vary between 1 and 4 , for a non cavitating computation.

\subsubsection{Numerical parameters}

For the non cavitating regime, computations are started from an uniform flowfield using a local time step. The numerical parameters used are :

- the CFL number, 10

- the preconditioned parameter, $K=1$ 
- Jacobi iterations for the implicit stage, 15

- the two coefficients of the artificial dissipation: $k^{(2)}=0$ and $k^{(4)}=0.032$

- the farfield value of turbulent viscosity $\mu_{t_{\infty}}=0.1 \mu_{\infty}$

All cavitating simulations are steady computations, which are started from the non cavitating numerical solution. The numerical parameters are:

- the vaporization pressure, $P_{v a p}=181100 \mathrm{~Pa}$ for $T_{\text {ref }}=293 \mathrm{~K}$ and $P_{\text {vap }}=$ $249880 \mathrm{~Pa}$ for $T_{\text {ref }}=303 \mathrm{~K}$

- the CFL number, 0.5

- the preconditioned parameter, $K=3$

- Jacobi iterations for the implicit stage, 15

- the two coefficients of the artificial dissipation: $k^{(2)}=0.5$ and $k^{(4)}=0.032$.

\subsection{The flat roof Venturi computations}

\section{Global analyses}

Different calculations were performed with and without thermal model (i.e. considering or not the thermal variations of the vapour pressure), for both EOS, summarized in Table 5. All computations used the Spalart-Allmaras turbulence model. The goal was to obtain a stable cavitation sheet with a length close to $80 \mathrm{~mm}$. The defined sheet length used for representing the computational results was determined by the length of the iso-line corresponding to a void ratio $\alpha=0.1$.

For the modified barotropic EOS and for three different $c_{b a r o}$ values, the numerical value of the inlet cavitation number is a little over-predicted in comparison with the experimental value close to 0.55. For the SG EOS without taking account thermodynamic effects (case 1), the cavity sheet obtained is 
short $\left(L_{c a v} \simeq 60 \mathrm{~mm}\right)$ but extremely thick and reaches the roof of the Venturi. From numerical tests done, we did not obtain a cavity sheet around $80 \mathrm{~mm}$ with this model.

\section{Local analyses}

Complementary analyses concern local void ratio profiles inside the cavity and the wall temperature depression. The experimental void ratio profiles are obtained for three stations, with a post processing algorithm from the signal of the optical probe [37]. The wall temperature depression is measured with three micro-thermocouples. The uncertainty on the temperature measurement is about $\pm 0.2 \mathrm{~K}$.

Figure 6 illustrates the numerical void ratio obtained with the stiffened gas EOS in comparison with the measurements. Without the thermal model, the cavity thickness is enormous and the maximum value of the void ratio is too small. The thermal model allows to improve the results by reducing the cavity thickness and increasing the void ratio. Yet, the thickness remains very high, there is a factor 3 with the experimental data.

The temperature deficit profile at station 1 is plotted in Fig. 7 for the stiffened gas EOS. As noticed for the void ratio profiles, the thermal model allows to improve the results : the wall temperature is in better agreement in comparison with the experimental data. The numerical wall temperature depression is about $1.68 \mathrm{~K}$ instead of $2.1 \mathrm{~K}$ for the measurement (the error is around $20 \%$ ).

Figures 8, 9 and 10 present the numerical void ratio obtained with the modified barotropic EOS for three values of the velocity $c_{\text {baro }}: 2.61 \mathrm{~m} / \mathrm{s}, 1.48 \mathrm{~m} / \mathrm{s}$ 
and $0.74 \mathrm{~m} / \mathrm{s}$, respectively. For all values of this parameter, the thermal model induces an increase of the cavity thickness and a decrease of the maximum value of the void ratio, in comparison with the computations without thermal model. We can verify that the void ratio values remain inside the convexity domain, given in Table 4 .

The influence of the velocity $c_{\text {baro }}$ on the void ratio profiles is showed in Fig. 11 for all computations accounting thermodynamic effects. As observed for cold water [12], the decrease of $c_{\text {baro }}$ reduces the cavity thickness. Moreover, the maximum void ratio value is augmented. At station 1, the numerical maximum is over-predicted in comparison with the experimental data. On the contrary, at station 3 , the peak value is under-estimated.

Figure 12 shows the temperature depression profile at station 1, obtained with and without thermal model, for the three values of $c_{\text {baro }}$. Without model, the peak of temperature deficit can reach large values. This maximum value is not localized at the wall but inside the cavity. Moreover, the decrease of the velocity $c_{\text {baro }}$ induces an augmentation of this maximum value. With the smallest value $c_{\text {baro }}=0.74 \mathrm{~m} / \mathrm{s}$, the peak of depression temperature is around $12 \mathrm{~K}$ instead of $2 \mathrm{~K}$ with the thermal model. For the wall temperature value, we observe the opposite effect: the decrease of $c_{\text {baro }}$ reduces this value. With the smallest $c_{\text {baro }}$ value, the wall temperature depression is near the experimental data. A very important temperature gradient in the cavity is obtained without the thermal model.

The influence of the velocity $c_{\text {baro }}$ on the temperature depression profile is showed in Fig. 15 for all computations accounting thermodynamic effects. The numerical wall values are in good agreement with the experimental data 
equal to $2.1 \mathrm{~K}$. With the smallest $c_{\text {baro }}$ value, the better result is obtained: the wall temperature deficit is $2.1 \mathrm{~K}$.

These first computations allow us to better evaluate the modified cavitation model including thermal variations. The stiffened gas EOS failed to predict thermodynamic effects. This cavitation model seems unappropriate for these computations.

On the contrary, the modified barotropic EOS can provide results in close agreement with the experimental data. The small non-equilibrium effect introduced in the model is enough to capture thermodynamic effects, especially with the smallest value of the parameter $c_{b a r o}$.

\section{Turbulence model influence}

A comparison of three turbulence models is proposed: the Spalart-Allmaras model (SA), the Jones-Launder $k-\varepsilon$ model (KE) and the Menter $k-\omega$ SST model (KWSST). All computations used the modified barotropic EOS with $c_{\text {baro }}=0.74 \mathrm{~m} / \mathrm{s}$. The goal was to obtain a stable cavitation sheet with a length close to $80 \mathrm{~mm}$.

The void ratio profiles obtained are plotted in Fig. 14. The Spalart-Allmaras and the Menter models give similar results both for the cavity thickness and the maximum void ratio, for the three stations. The void ratio profiles provided by the Jones-Launder model present numerical oscillations, especially on the top of the cavity. The cavity thickness is in better agreement with the experimental data. The maximum value of the void ratio is similar to those obtained by other turbulence models. 
The temperature deficit profile is showed in Fig. 15. As remarked previously, the Spalart-Allmaras and the Menter models give very close results, and the Jones-Launder model provides spurious oscillations on the top of the cavity. The convergence with the $k-\varepsilon$ model was not rigourously obtained in spite of the use of high values of artificial dissipation, maybe due to an unsteady behaviour.

\subsection{The undulated roof Venturi computations}

For all these computations, only the modified barotropic EOS is considered with $c_{\text {baro }}=0.74 \mathrm{~m} / \mathrm{s}$ and coupled with the Spalart-Allmaras turbulence model. Two reference temperatures were tested.

Reference temperature $T_{\text {ref }}=293 \mathrm{~K}$

Different calculations were performed by considering four inlet velocities $V_{\text {inlet }}$, three $\sigma_{\text {inlet }}$ values, for a reference temperature $T_{r e f}=293 \mathrm{~K}$. The goal was to obtain a stable cavitation sheet with a length $L_{\text {cav }}$ close to the experimental value. The experimental data and the numerical results are summarized in Table 6.

For all computations, the numerical value of the inlet cavitation number is under-predicted in comparison with the experimental value. The gap varies between 0.05 and 0.12 . Moreover, higher is the inlet velocity, more a small variation on $\sigma_{\text {inlet }}$ has an influence on the cavity length.

The numerical and experimental temperature depressions at station 1 are given in Table 7. For the lower inlet velocity, the numerical results are in very close agreement with the experimental data (the error is less than $1 \%$ ). But, 
higher is the inlet velocity, higher is the error. For the highest velocity (cases 10, 11 and 12), a large discrepancy is observed: the temperature deficit is overestimated on about 1K. Except for this velocity, the model provides a very good quality of results based on the temperature depression (the maximum error is around $5 \%$ ). Maybe, a higher value of the parameter $c_{b a r o}$ could improve the results.

The difficulties to capture thermodynamic effects at high inlet velocities were also remarked by Rolland [32] with another homogeneous equilibrium model. The metastability of the fluid becomes important and equilibrium assumptions are too stiff.

Reference temperature $T_{\text {ref }}=303 \mathrm{~K}$

Different calculations were performed by considering three $\sigma_{\text {inlet }}$ values for an inlet velocity $V_{\text {inlet }}=22 \mathrm{~m} / \mathrm{s}$ and a reference temperature $T_{r e f}=303 \mathrm{~K}$. The freon R-114 characteristics are given in Tables 1 and 2 at this temperature. The goal was to obtain a stable cavitation sheet with a length close to the experimental one. The experimental data and the numerical results are summarized in Table 8. As noticed previously, the numerical $\sigma_{\text {inlet }}$ values are largely smaller in comparison with the experimental data.

The wall temperature depression is plotted in Fig. 16 versus the distance $\left(x-x_{\text {throat }}\right) / L_{\text {cav }}$ for the three inlet cavitation numbers considered. For the cases 1 and 2, the temperature deficit is well predicted by computations on the first part of the cavity. At the end of the cavity, a large discrepancy is observed (around $1.5 \mathrm{~K}$ ). For the case 3 , the numerical results are in very close agreement with the experimental data, in all the cavity. The analysis of results concerning the closure part of the cavity is complex because of possible unsteadiness due to a re-entrant jet. In this case, the present stationary computations are unable 
to correctly describe the flow behaviour.

\section{Conclusion}

A two-phase one-fluid compressible viscous CFD method was presented and applied to Venturi R-114 cavitating flows. Several numerical aspects of the method were outlined, including a low Mach number preconditioner, a lowstorage implicit strategy and preconditioned characteristic relations for the boundary conditions treatment. Two different cavitation models based on two mixture EOS were tested and compared. The respect of the second principle of thermodynamic was verified through the convexity conditions.

Steady results of the temperature depression and void ratio profiles were presented for Venturi geometries, with different model coefficients. The mixture EOS based on the stiffened gas law was not able to predict a cavity in close agreement with the experimental data. It is certainly due to the equilibrium assumptions made. On the other hand, the modified barotropic model made it possible to accurately simulate the temperature drop in such cavitating

flows, especially with the smallest value of the parameter $c_{\text {baro }}$. This model introduces a small non-equilibrium effect, which is enough to capture thermodynamic effects, as much as the inlet velocity remains moderate. For higher velocities, the model fails to predict the thermal depression (in comparison with the experimental data, the error is around 50\%).

Finally, a comparison between three transport-equation turbulence models were proposed. Similar solutions were obtained with Spalart-Allmaras and Menter $k-\omega$ SST models. The Jones-Launder $k-\varepsilon$ model would seem more sensitive to the flow unsteadiness, generating numerical oscillations.

Additional works are in progress to simulate cavitating flows with cryogenic fluids (liquid hydrogen), to develop relaxed models (HRM) and to pursue com- 
parative analyses between numerical approaches, turbulence and cavitation models. Moreover, further experimental works concerning local measurements in thermosensible fluid cavitation are capital to allow model calibration and validation.

\section{Appendix}

Appendix $A$ : the preconditioned system for pure phases

For pure phases which follow the stiffened gas EOS, the different matrices are:

$$
\begin{aligned}
& R=\frac{\partial W}{\partial w}=\left[\begin{array}{ccc}
\frac{(\gamma-1)}{2} u^{2} & -(\gamma-1) u & (\gamma-1) \\
-u / \rho & 1 / \rho & 0 \\
\frac{u^{2}-E-q}{\rho} & -\frac{u}{\rho} & \frac{1}{\rho}
\end{array}\right] \\
& R^{-1}=\frac{\partial w}{\partial W}=\left[\begin{array}{ccc}
\frac{1}{(\gamma-1)(e-q)} & 0 & -\frac{\rho}{e-q} \\
\frac{u}{(\gamma-1)(e-q)} & \rho & -\frac{\rho u}{e-q} \\
\frac{E-q}{(\gamma-1)(e-q)} & \rho u & -\frac{\rho u^{2}}{2(e-q)}
\end{array}\right]
\end{aligned}
$$


Appendix $B$ : the speed of sound with a mixture of stiffened gas

Starting from the usual thermodynamic relation

$$
d e=T d s+\frac{P}{\rho^{2}} d \rho \quad \text { or } \quad d(\rho e)=\rho T d s+h d \rho
$$

And with the differential of $\rho e$ :

$$
d(\rho e)=\left(\frac{\partial \rho e}{\partial \rho}\right)_{P} d \rho+\left(\frac{\partial \rho e}{\partial P}\right)_{\rho} d P
$$

We can obtained the differential of the pressure $P$ :

$$
\left(\frac{\partial \rho e}{\partial P}\right)_{\rho} d P=\rho T d s+\left[h-\left(\frac{\partial \rho e}{\partial \rho}\right)_{P}\right] d \rho
$$

We deduce an expression of the speed of sound:

$$
c^{2}=\left(\frac{\partial P}{\partial \rho}\right)_{s}=\frac{h-\left(\frac{\partial \rho e}{\partial \rho}\right)_{P}}{\left(\frac{\partial \rho e}{\partial P}\right)_{\rho}}
$$

We assume that phases are in thermodynamic equilibrium then the pressure $P$ and the temperature $T$ are linked by a relation. Therefore we have $\left(\frac{\partial P}{\partial \rho}\right)_{T}=0,\left(\frac{\partial T}{\partial \rho}\right)_{P}=0$ and $\left(\frac{\partial P}{\partial T}\right)_{\rho}=\frac{d P_{v a p}}{d T}=\frac{d P}{d T}$ is constant.

The derivative of the void ratio $\alpha$ with the density and the pressure have to be evaluated :

$$
\begin{aligned}
& \left(\frac{\partial \alpha}{\partial \rho}\right)_{P}=\frac{-1}{\rho_{L}-\rho_{V}} \\
& \left(\frac{\partial \alpha}{\partial P}\right)_{\rho}=\frac{-1}{\rho_{V}-\rho_{L}} \frac{d T}{d P}\left[\alpha \frac{d \rho_{V}}{d T}+(1-\alpha) \frac{d \rho_{L}}{d T}\right]
\end{aligned}
$$

With the stiffened gas EOS, we have the following equalities: 


$$
\begin{aligned}
\left(\frac{\partial \rho e}{\partial P}\right)_{\rho} & =\alpha\left(\frac{\partial \rho_{V} e_{V}}{\partial P}\right)_{\rho}+(1-\alpha)\left(\frac{\partial \rho_{L} e_{L}}{\partial P}\right)_{\rho}+\left(\rho_{V} h_{V}-\rho_{L} h_{L}\right)\left(\frac{\partial \alpha}{\partial P}\right)_{\rho} \\
& =\frac{1}{\gamma-1}+\frac{\rho_{V} h_{V}-\rho_{L} h_{L}}{\rho_{L}-\rho_{V}} \frac{d T}{d P}\left[\alpha \frac{d \rho_{V}}{d T}+(1-\alpha) \frac{d \rho_{L}}{d T}\right] \\
\left(\frac{\partial \rho e}{\partial \rho}\right)_{P} & =\frac{\partial}{\partial \rho}\left[\alpha\left(\frac{P}{\gamma_{V}-1}+\rho_{V} q_{V}+\frac{\gamma_{V}}{\gamma_{V}-1} P_{\infty}^{V}\right)+(1-\alpha)\left(\frac{P}{\gamma_{L}-1}+\rho_{L} q_{L}+\frac{\gamma_{L}}{\gamma_{L}-1} P_{\infty}^{L}\right)\right] \\
& =\frac{\rho_{L} h_{L}-\rho_{V} h_{V}}{\rho_{L}-\rho_{V}}
\end{aligned}
$$

Finally, the speed of sound is:

$$
\rho c^{2}=\frac{1}{\left(\frac{\partial \rho e}{\partial P}\right)_{\rho}}\left[\frac{\rho_{V} \rho_{L}}{\left(\rho_{L}-\rho_{V}\right)}\left(h_{V}-h_{L}\right)\right]
$$

Appendix $C: g$ and $\Gamma$ parameters for the mixture of stiffened gas EOS

Starting from thermodynamic relations and differentials of $\rho e$ and $\rho h$ :

$$
\begin{aligned}
d(\rho e) & =\rho T d s+h d \rho \\
d(\rho h) & =d(\rho e)+d P \\
d(\rho e) & =\left(\frac{\partial \rho e}{\partial \rho}\right)_{P} d \rho+\left(\frac{\partial \rho e}{\partial P}\right)_{\rho} d P \\
d(\rho h) & =\left(\frac{\partial \rho h}{\partial T}\right)_{\rho} d T+\left(\frac{\partial \rho h}{\partial \rho}\right)_{T} d \rho
\end{aligned}
$$

We can obtained the differential of temperature $d T$ :

$$
\left(\frac{\partial \rho h}{\partial T}\right)_{\rho} d T=\rho T\left[\frac{\left(\frac{\partial \rho e}{\partial \rho}\right)_{P}+1}{\left(\frac{\partial \rho e}{\partial \rho}\right)_{P}}\right] d s-\left[\left(\frac{\partial \rho h}{\partial \rho}\right)_{T}-h-c^{2}\right] d \rho
$$

We deduce these equalities:

$$
\left(\frac{\partial T}{\partial s}\right)_{\rho}=\frac{\rho T}{\left(\frac{\partial \rho h}{\partial T}\right)_{\rho}}\left[\frac{\left(\frac{\partial \rho e}{\partial \rho}\right)_{P}+1}{\left(\frac{\partial \rho e}{\partial \rho}\right)_{P}}\right] \quad ; \quad\left(\frac{\partial T}{\partial \rho}\right)_{s}=\frac{h-\left(\frac{\partial \rho h}{\partial \rho}\right)_{T}+c^{2}}{\left(\frac{\partial \rho h}{\partial T}\right)_{\rho}}(71)
$$

With the stiffened gas EOS, we have: 


$$
\begin{aligned}
& \left(\frac{\partial \rho h}{\partial T}\right)_{\rho}=\rho C_{p}+\left[\frac{\left(C_{p_{L}}-C_{p_{V}}\right) T+\left(q_{L}-q_{V}\right)}{\rho_{V}-\rho_{L}}\right]\left[\alpha \rho_{L} \frac{d \rho_{V}}{d T}+(1-\alpha) \rho_{V} \frac{d \rho_{L}}{d T}\right] \\
& \left(\frac{\partial \rho h}{\partial \rho}\right)_{T}=\frac{\left(\rho_{V} C_{p_{V}}-\rho_{L} C_{p_{L}}\right) T+\rho_{V} q_{V}-\rho_{L} q_{L}}{\rho_{V}-\rho_{L}}
\end{aligned}
$$

Finally the expression of the dimensionless parameters are:

$$
\begin{aligned}
& g=\frac{P}{\rho T^{2}}\left(\frac{\partial T}{\partial s}\right)_{\rho}=\frac{P\left(1+\left(\frac{\partial \rho e}{\partial \rho}\right)_{P}\right)}{T\left(\frac{\partial \rho e}{\partial \rho}\right)_{P}\left(\frac{\partial \rho h}{\partial T}\right)_{\rho}} \\
& \Gamma=\frac{\rho}{T}\left(\frac{\partial T}{\partial \rho}\right)_{s}=\frac{\rho}{P}\left(\frac{\partial \rho e}{\partial \rho}\right)_{P} g c^{2}
\end{aligned}
$$

Appendix D : the speed of sound with the modified barotropic EOS

Starting from the usual thermodynamic relation

$$
d(\rho e)=\rho T d s+h d \rho
$$

And with the differential of $\rho h$ :

$$
d(\rho h)=\left(\frac{\partial \rho h}{\partial \rho}\right)_{P} d \rho+\left(\frac{\partial \rho h}{\partial P}\right)_{\rho} d P
$$

We can obtained the differential of the pressure $P$ :

$$
\left[\left(\frac{\partial \rho h}{\partial P}\right)_{\rho}-1\right] d P=\rho T d s+\left[h-\left(\frac{\partial \rho h}{\partial \rho}\right)_{P}\right] d \rho
$$

We deduce an expression of the speed of sound:

$$
c^{2}=\left(\frac{\partial P}{\partial \rho}\right)_{s}=\frac{h-\left(\frac{\partial \rho h}{\partial \rho}\right)_{P}}{\left(\frac{\partial \rho h}{\partial P}\right)_{\rho}-1}
$$

This EOS allows to introduce a small non-equilibrium thermodynamic effect. Indeed, we have no more $\left(\frac{\partial T}{\partial \rho}\right)_{P}=0$ and $\left(\frac{\partial P}{\partial \rho}\right)_{T}=0$ but new expressions : 


$$
\begin{aligned}
& \left(\frac{\partial \rho}{\partial T}\right)_{P}=-\frac{d P}{d T} \frac{1}{A c_{\text {baro }}^{2}} \cos \left(\frac{P-P_{\text {vap }}(T)}{0.5\left(\rho_{L}^{\text {sat }}-\rho_{V}^{\text {sat }}\right) c_{\text {baro }}^{2}}\right) \\
& \left(\frac{\partial \rho}{\partial P}\right)_{T}=\frac{1}{A c_{\text {baro }}^{2}} \cos \left(\frac{P-P_{\text {vap }}(T)}{0.5\left(\rho_{L}^{\text {sat }}-\rho_{V}^{\text {sat }}\right) c_{\text {baro }}^{2}}\right) \\
& \left(\frac{\partial P}{\partial T}\right)_{\rho}=-\left(\frac{\partial P}{\partial \rho}\right)_{T}\left(\frac{\partial \rho}{\partial T}\right)_{P}=\frac{d P}{d T}
\end{aligned}
$$

With this EOS, we have the following equalities:

$$
\begin{aligned}
& \left(\frac{\partial \rho h}{\partial P}\right)_{\rho}=\rho C_{p}\left(\frac{\partial T}{\partial P}\right)_{\rho}=\frac{d T}{d P} \rho C_{p} \\
& \left(\frac{\partial \rho h}{\partial \rho}\right)_{P}=\rho C_{p}\left(\frac{\partial T}{\partial \rho}\right)_{P}+\left[\frac{\left(\rho_{L} C_{p_{L}}-\rho_{V} C_{p_{V}}\right) T+\left(\rho_{L} q_{L}-\rho_{V} q_{V}\right)}{\rho_{L}-\rho_{V}}\right]
\end{aligned}
$$

Finally, the speed of sound is :

$$
c^{2}=\frac{\frac{\rho_{V} \rho_{L}}{\rho\left(\rho_{L}-\rho_{V}\right)}\left(h_{V}-h_{L}\right)-\rho C_{p}\left(\frac{\partial T}{\partial \rho}\right)_{P}}{\rho C_{p} \frac{d T}{d P}-1}
$$

Appendix $E: g$ and $\Gamma$ parameters for the modified barotropic EOS

Starting from thermodynamic relations and differentials of $\rho h$ :

$$
\begin{aligned}
d(\rho e) & =\rho T d s+h d \rho \\
d(\rho h) & =d(\rho e)+d P \\
d(\rho h) & =\left(\frac{\partial \rho h}{\partial \rho}\right)_{P} d \rho+\left(\frac{\partial \rho h}{\partial P}\right)_{\rho} d P \\
d(\rho h) & =\left(\frac{\partial \rho h}{\partial T}\right)_{\rho} d T+\left(\frac{\partial \rho h}{\partial \rho}\right)_{T} d \rho
\end{aligned}
$$

We can obtained the differential of temperature $d T$ :

$$
\left(\frac{\partial \rho h}{\partial T}\right)_{\rho} d T=\rho T\left[\frac{\left(\frac{\partial \rho h}{\partial P}\right)_{\rho}}{\left(\frac{\partial \rho h}{\partial P}\right)_{\rho}-1}\right] d s-\left[\left(\frac{\partial \rho h}{\partial \rho}\right)_{T}-h-c^{2}\right] d \rho
$$


We deduce these equalities:

$$
\left(\frac{\partial T}{\partial s}\right)_{\rho}=\frac{\rho T}{\left(\frac{\partial \rho h}{\partial T}\right)_{\rho}}\left[\frac{\left(\frac{\partial \rho h}{\partial P}\right)_{\rho}}{\left(\frac{\partial \rho h}{\partial P}\right)_{\rho}-1}\right] \quad ; \quad\left(\frac{\partial T}{\partial \rho}\right)_{s}=\frac{h-\left(\frac{\partial \rho h}{\partial \rho}\right)_{T}+c^{2}}{\left(\frac{\partial \rho h}{\partial T}\right)_{\rho}}
$$

With this EOS, we have:

$$
\begin{aligned}
\left(\frac{\partial \rho h}{\partial T}\right)_{\rho} & =\rho C_{p} \\
\left(\frac{\partial \rho h}{\partial \rho}\right)_{T} & =\frac{\left(\rho_{L} C_{p_{L}}-\rho_{V} C_{p_{V}}\right) T+\rho_{L} q_{L}-\rho_{V} q_{V}}{\rho_{L}-\rho_{V}} \\
h-\left(\frac{\partial \rho h}{\partial \rho}\right)_{T}+c^{2} & =\rho C_{P}\left[\frac{d T}{d P} c^{2}+\left(\frac{\partial T}{\partial \rho}\right)_{P}\right]
\end{aligned}
$$

Finally the expression of the dimensionless parameters is:

$$
\begin{aligned}
g & =\frac{P}{\rho T^{2}}\left(\frac{\partial T}{\partial s}\right)_{\rho}=\frac{P}{T} \frac{1}{\rho C_{p}-\frac{d P}{d T}} \\
\Gamma & =\frac{\rho}{T}\left(\frac{\partial T}{\partial \rho}\right)_{s}=\frac{\rho c^{2}}{T \frac{d P}{d T}}+\frac{\rho}{P}\left(\frac{\partial T}{\partial \rho}\right)_{P}
\end{aligned}
$$

Assuming the cosine value is equal to 1 in the expression of $\left(\frac{\partial T}{\partial \rho}\right)_{P}$ (that is to say the pressure is equal to $P_{v a p}(T)$ ). The expression of $\Gamma$ becomes :

$$
\Gamma=\frac{\rho}{T \frac{d P}{d T}}\left(c^{2}-c_{\text {baro }}^{2}\right)
$$




\section{References}

[1] Ahuja V, Hosangadi A. A numerical study of cavitation in cryogenic fluids, part I: mean flow parametric studies. Proceedings of sixth International Symposium on Cavitation CAV2006, Wageningen, The Netherlands, September 11-15 2006.

[2] Barret M, Faucher E, Herard JM. Schemes to compute unsteady flashing flows. AIAA Journal 2002;40(5):905-913.

[3] Downar-Zapolski P, Bilicki Z, Bolle L, Franco J. The non-equilibrium relaxation model for one-dimensional flashing liquid flow. Int. Journal of Multiphase Flow $1996 ; 22(3): 473-483$.

[4] Bilicki Z, Kwidzinski R, Mohammadein SA. Evaluation of the relaxation time of heat and mass exchange in the liquid-vapour bubble flow. Int. Journal of Heat and Mass Transfer 1996;39(4):753-759.

[5] Billet ML, Holl JW, Weir DS. Correlations of thermodynamic effects for developped cavitation. Journal of Fluids Engineering 1981;103(12):534-542.

[6] Choi YH, Merkle CL. The application of preconditioning to viscous flows. Journal of Computational Physics 1993;105(2):207-223.

[7] Clerc S. Numerical simulation of the homogeneous equilibrium model for twophase flows. Journal of Computational Physics 2000;161(1):354-375.

[8] Cooper P. Analysis of single and two-phase flow in turbopump inducers. Journal of Engineering Power 1967;89:577-588.

[9] Delannoy Y, Kueny J-L. Two phase flow approach in unsteady cavitation modelling. In: Cavitation and Multiphase Flow Forum, ASME-FED 1990;98:153-158.

[10] Edwards JR, Franklin RK. Low-Diffusion Flux Splitting methods for real fluid flows with phase transition. AIAA Journal 2000;38(9):1624-1633.

[11] Fruman DH, Reboud J-L, Stutz B. Estimation of thermal effects in cavitation 
of thermosensible liquids. Int. Journal of Heat and Mass Transfer 1999;42:31953204 .

[12] Goncalvès E, Fortes Patella R. Numerical Simulation of Cavitating Flows with Homogeneous Models. Computers \& Fluids 2009, accepted.

[13] Guillard H, Viozat C. On the behaviour of upwind schemes in the low Mach number limit. Computers \& Fluids 1999;28(1):63-86.

[14] Harten A, Lax PD, Lervermore CD, Morokoff W. Convex entropies and hyperbolic ity for general euler equations. SIAM Journal on Numerical Analysis 1998;35(6):2117-2127.

[15] Helluy P, Seguin N. Relaxation models of phase transition flows. Mathematical Modelling and Numerical Analysis 2006;40(2):331-352.

[16] Holl JW, Billet ML, Weir DS. Thermodynamic effects on developed cavitation. Journal of Fluids Engineering 1975;97:507-514.

[17] Hord J. Cavitation in liquid Cryogens, Vol. 4, combined correlations for Venturi, Hydrofoil, Ogives and Pumps. NASA CR-2448, 1974.

[18] Hosangadi A, Ahuja V. Numerical study of cavitation in cryogenic fluids. Journal of Fluids Engineering 2005;127(2):267-281.

[19] Jameson A, Schmidt W, Turkel E. Numerical simulation of the Euler equations by finite volume method using Runge-Kutta time stepping schemes. AIAA Paper 81-1259; In: 14th Fluid and Plasma Dynamics Conference, Palo Alto, California; June 1981.

[20] Jones WP, Launder BE. The Prediction of Laminarization with a Two-Equation Model of Turbulence. Int. Journal of Heat and Mass Transfer 1972;15:301-314.

[21] Le Metayer O, Massoni J, Saurel R. Elaborating equations of state of a liquid and its vapor for two-phase flow models. Int. Journal of Thermal Sciences 2004;43:265-276.

[22] Le Metayer O, Massoni J, Saurel R. Modelling evaporation fronts with reactive Riemann solvers. Journal of Computational Physics 2005;205(2):567-610. 
[23] Luo H, Baum JD, Lohner R. A fast, matrix-free implicit method for compressible flows on unstructured grids. Journal of Computational Physics 1998;146(2):664-690.

[24] Menter FR. Two-equation eddy-viscosity turbulence models for engineering applications. AIAA Journal 1994;32(8):1598-1605.

[25] Merci B, Steelant J, Vierendeels J, Riemslagh K, Dick E. Computational treatment of source terms in two-equation turbulence models. AIAA Journal 2000;38(11):2085-2093.

[26] Merle L. Etude expérimentale et modèle physique d'un écoulement cavitant avec effet thermodynamique. Ph.D. Thesis, Grenoble Polytechnic Institute, 1994.

[27] Moore RD, Ruggeri RS. Prediction of thermodynamic effects on developed cavitation based on liquid hydrogen and freon 114 data in scaled venturis. NASA TN D-4899, November 1968.

[28] Perrier V. The Chapman-Jouguet closure for the Riemann problem with vaporization. SIAM Journal On Applied Mathematics 2008;68(5):1333-1359.

[29] Rapposelli E, d'Agostino L. A barotropic cavitation model with thermodynamic effects. Proceedings of fifth International Symposium on Cavitation CAV2003, Osaka, Japan, November 1-4 2003.

[30] Roe PL. Approximate Riemann solvers, parameters vectors, and difference schemes. Journal of Computational Physics 1981;43:357-372.

[31] Rolland J, Fortes Patella R, Goncalves E, Boitel G, Barre S. Experiments and modelling of cavitating flows in Venturi, Part I: stable cavitation. Proceedings of sixth International Symposium on Cavitation CAV2006, Wageningen, The Netherlands, September 11-15 2006.

[32] Rolland J. Modélisation des écoulements cavitants dans les inducteurs de turbopompes : prise en compte des effets thermodynamiques. Ph.D. Thesis, Grenoble Polytechnic Institute, 2008. 
[33] Saurel R, Le Metayer O. A multiphase model for compressible flows with interfaces, shocks, detonation waves and cavitation. Journal of Fluid Mechanics 2001;431:239-271.

[34] Saurel R, Petitpas F. Abgrall R. Modelling phase transition in metastable liquids: application to cavitating and flashnig flows. Journal of Fluid Mechanics 2008;607:313-350.

[35] Spalart PR, Allmaras SR. A one-equation turbulence model for aerodynamic flows. La Recherche Aerospatiale 1994;1:5-21.

[36] Stahl HA, Stepanoff AJ, Phillipsburg NJ. Thermodynamic aspects of cavitation in centrifugal pumps. ASME Journal of Basic Engineering 1956;78:1691-1693.

[37] Stutz B. Analyse de la structure diphasique et instationnaire de poches de cavitation. Ph.D. Thesis, Grenoble Polytechnic Institute, 1996.

[38] Tatsumi S, Martinelli L, Jameson A. Flux-Limited Schemes for the Compressible Navier-Stokes Equations. AIAA Journal 1995;33(2):252-261.

[39] Turkel E. Preconditioned methods for solving the incompressible and low speed compressible equations. Journal of Computational Physics 1987;172(2):277-298.

[40] Utturkar Y, Wu J, Wang G, Shyy W. Recent progress in modelling of cryogenic cavitation for liquid rocket propulsion. Progress in Aerospace Sciences 2005;41:558-608.

[41] Wallis G. One-dimensional two-phase flow. New York: McGraw-Hill; 1967.

[42] Zhang XB, Qiu LM, Gao Y, Zhang XJ. Computational fluid dynamic study on cavitation in liquid nitrogen. Cryogenics 2008;48(9-10):432-438. 
Table 1

Characteristics of water and freon R-114 at saturation.

\begin{tabular}{ccccccc} 
fluid & $T_{\text {ref }}(\mathrm{K})$ & $\rho_{L}\left(\mathrm{~kg} / \mathrm{m}^{3}\right)$ & $\rho_{V}\left(\mathrm{~kg} / \mathrm{m}^{3}\right)$ & $P_{\text {vap }}(\mathrm{Pa})$ & $\frac{d P_{\text {vap }}}{d T}(\mathrm{~Pa} / \mathrm{K})$ & $\Delta T^{*}(\mathrm{~K})$ \\
\hline water & 293 & 998 & 0.02 & 2339 & 143 & 0.01 \\
R-114 & 293 & 1470.3 & 13.52 & 181100 & 6000 & 1.22 \\
R-114 & 303 & 1439.6 & 18.36 & 249880 & 7700 & 1.61 \\
\hline
\end{tabular}


Table 2

Parameters of the stiffened gas EOS, freon R-114.

\begin{tabular}{llllllll} 
& $T_{\text {ref }}(\mathrm{K})$ & $\gamma$ & $P_{\infty}(\mathrm{Pa})$ & $\mathrm{q}(\mathrm{J} / \mathrm{kg})$ & $C_{p}(\mathrm{~J} / \mathrm{K} . \mathrm{kg})$ & $c(\mathrm{~m} / \mathrm{s})$ & $\rho\left(\mathrm{kg} / \mathrm{m}^{3}\right)$ \\
\hline liquid & 293 & 1.1 & $3.83510^{7}$ & $-0.6910^{5}$ & 984 & 170 & 1470.3 \\
vapour & 293 & 1.07 & 0 & $0.14210^{6}$ & 700 & 120 & 13.52 \\
liquid & 303 & 1.1 & $3.9410^{7}$ & $-0.7410^{5}$ & 1000 & 174 & 1439.6 \\
& & & & & & & \\
vapour & 303 & 1.07 & 0 & $0.13610^{6}$ & 724 & 120 & 18.36 \\
\hline
\end{tabular}


Table 3

Parameters of thermal variations for the stiffened gas EOS.

\begin{tabular}{llll}
\hline$T_{\text {ref }}(\mathrm{K})$ & $\frac{d P_{\text {vap }}}{d T}(\mathrm{~Pa} / \mathrm{K})$ & $\frac{d \rho_{L}}{d T}\left(\mathrm{~kg} / \mathrm{m}^{3} \cdot \mathrm{K}\right)$ & $\frac{d \rho_{V}}{d T}\left(\mathrm{~kg} / \mathrm{m}^{3} \cdot \mathrm{K}\right)$ \\
\hline 293 & 6000 & -3.0 & 0.417 \\
303 & 7700 & -3.1 & 0.531 \\
\hline
\end{tabular}


Table 4

Parameters of the modified barotropic EOS, freon R-114.

\begin{tabular}{llllll}
$T_{\text {ref }}(\mathrm{K})$ & $\rho_{L}^{\text {sat }}\left(\mathrm{kg} / \mathrm{m}^{3}\right)$ & $\rho_{V}^{\text {sat }}\left(\mathrm{kg} / \mathrm{m}^{3}\right)$ & $c_{\text {baro }}(\mathrm{m} / \mathrm{s})$ & $\Delta P(\mathrm{~Pa})$ & convexity domain \\
\hline 293 & 1470.6 & 13.52 & 2.61 & 7794 & $\alpha<0.9$ \\
293 & 1470.4 & 13.52 & 1.48 & 2551 & $\alpha<0.8$ \\
293 & 1470.33 & 13.52 & 0.74 & 638 & $\alpha<0.7$ \\
303 & 1439.62 & 18.36 & 0.74 & 638 & $\alpha<0.7$ \\
\hline
\end{tabular}


Table 5

The different computations, flat roof Venturi.

\begin{tabular}{llllll} 
case & EOS & thermal model & $c_{\text {baro }}(\mathrm{m} / \mathrm{s})$ & $\sigma_{\text {inlet }}$ & $L_{\text {cav }}(\mathrm{mm})$ \\
\hline 1 & SG & without & - & 0.44 & 60 \\
2 & SG & with & - & 0.54 & 82 \\
3 & baro & without & 2.61 & 0.60 & 76 \\
4 & baro & with & 2.61 & 0.60 & 75 \\
5 & baro & without & 1.48 & 0.58 & 75 \\
6 & baro & with & 1.48 & 0.57 & 75 \\
7 & baro & without & 0.74 & 0.58 & 78 \\
8 & baro & with & 0.74 & 0.57 & 75 \\
\hline
\end{tabular}


Table 6

Parameters of the different computations, $T_{r e f}=293 \mathrm{~K}$, undulated roof Venturi.

\begin{tabular}{|c|c|c|c|c|c|}
\hline \multirow[b]{2}{*}{ case } & \multicolumn{3}{|l|}{ experiment } & \multicolumn{2}{|c|}{ computations } \\
\hline & $V_{\text {inlet }}(\mathrm{m} / \mathrm{s})$ & $\sigma_{\text {inlet }}$ & $L_{\text {cav }}(\mathrm{mm})$ & $\sigma_{\text {inlet }}$ & $L_{\text {cav }}(\mathrm{mm})$ \\
\hline 1 & 14.4 & 0.68 & 30 & 0.56 & 28 \\
\hline 2 & 14.4 & 0.61 & 43 & 0.54 & 39 \\
\hline 3 & 14.4 & 0.55 & 67 & 0.50 & 69 \\
\hline 4 & 18 & 0.61 & 47 & 0.54 & 43 \\
\hline 5 & 18 & 0.56 & 63 & 0.51 & 64 \\
\hline 6 & 18 & 0.54 & 88 & 0.495 & 88 \\
\hline 7 & 27 & 0.62 & 55 & 0.54 & 55 \\
\hline 8 & 27 & 0.58 & 75 & 0.535 & 74 \\
\hline 9 & 27 & 0.57 & 96 & 0.525 & 99 \\
\hline 10 & 38.2 & 0.63 & 64 & 0.543 & 68 \\
\hline 11 & 38.2 & 0.60 & 84 & 0.540 & 82 \\
\hline 12 & 38.2 & 0.59 & 103 & 0.522 & 97 \\
\hline
\end{tabular}




\section{Table 7}

Temperature depression, $T_{r e f}=293 \mathrm{~K}$, undulated roof Venturi.

experiment computations

\begin{tabular}{|c|c|c|c|c|}
\hline case & $T_{r e f}-T(\mathrm{~K})$ & $T_{r e f}-T(\mathrm{~K})$ & abs. error $(\mathrm{K})$ & relat. error $(\%)$ \\
\hline 1 & 2.1 & 2.09 & 0.01 & 0.5 \\
\hline 2 & 2.29 & 2.31 & 0.02 & 0.9 \\
\hline 3 & 2.54 & 2.56 & 0.02 & 0.8 \\
\hline 4 & 2.31 & 2.37 & 0.06 & 2.6 \\
\hline 5 & 2.62 & 2.62 & 0. & 0. \\
\hline 6 & 2.81 & 2.72 & 0.09 & 3.2 \\
\hline 7 & 2.12 & 2.23 & 0.11 & 5.2 \\
\hline 8 & 2.48 & 2.51 & 0.03 & 1.2 \\
\hline 9 & 2.75 & 2.78 & 0.03 & 1.1 \\
\hline 10 & 2.01 & 2.92 & 0.91 & 45 \\
\hline 11 & 2.18 & 3.10 & 0.92 & 42 \\
\hline 12 & 2.51 & 3.86 & 1.35 & 54 \\
\hline
\end{tabular}


Table 8

Parameters of the different computations, $T_{r e f}=303 \mathrm{~K}$, undulated roof Venturi.

\begin{tabular}{lllll} 
& & & & \\
& & & & \\
casperiment & $\sigma_{\text {inlet }}$ & $L_{\text {cav }}(\mathrm{mm})$ & $\sigma_{\text {inlet }}$ & $L_{\text {cav }}(\mathrm{mm})$ \\
\hline 1 & 0.67 & 35 & 0.56 & 30 \\
2 & 0.59 & 50 & 0.50 & 50 \\
3 & 0.55 & 66 & 0.485 & 62 \\
\hline
\end{tabular}




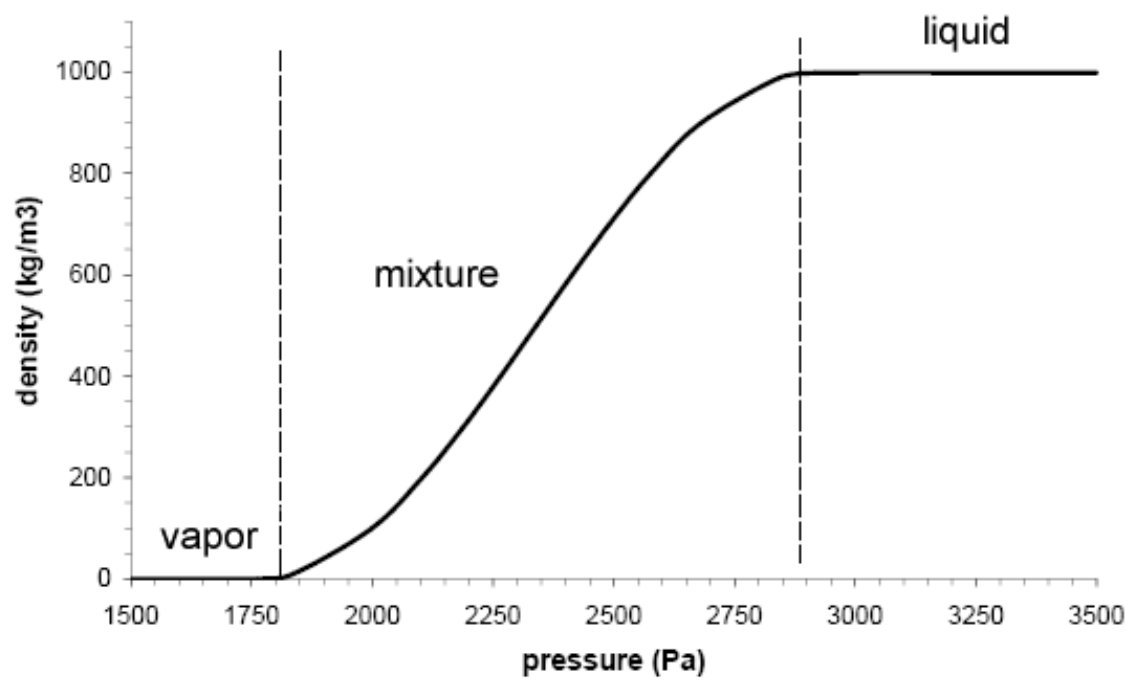

Fig. 1. The sinusoidal barotropic EOS for cold water. 


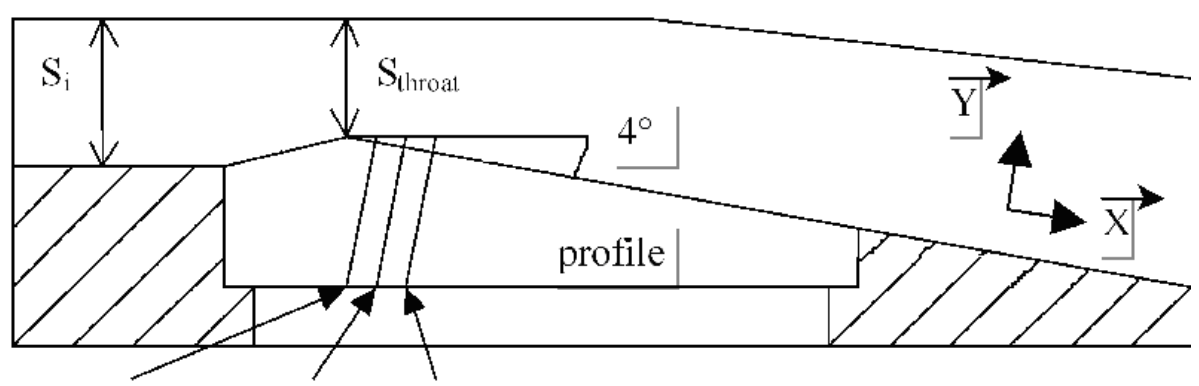

Station 1 Station 2 Station 3

Fig. 2. Schematic view of the Venturi profile. 


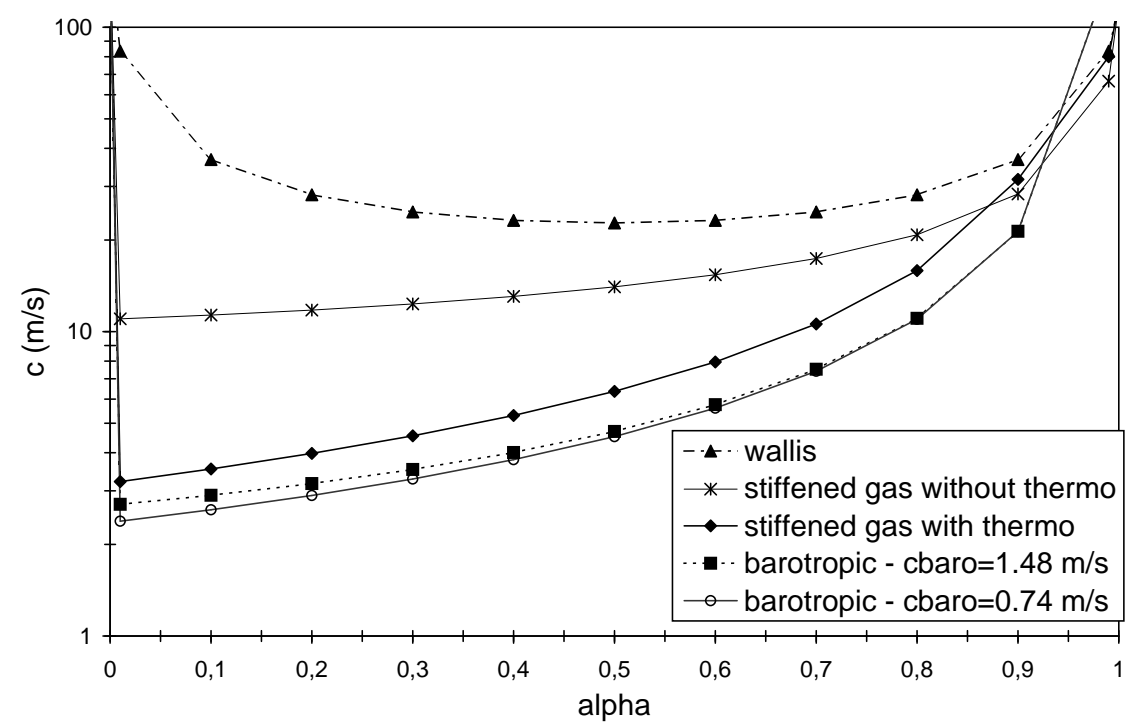

Fig. 3. Speed of sound for different EOS, $T_{r e f}=293 \mathrm{~K}$. 


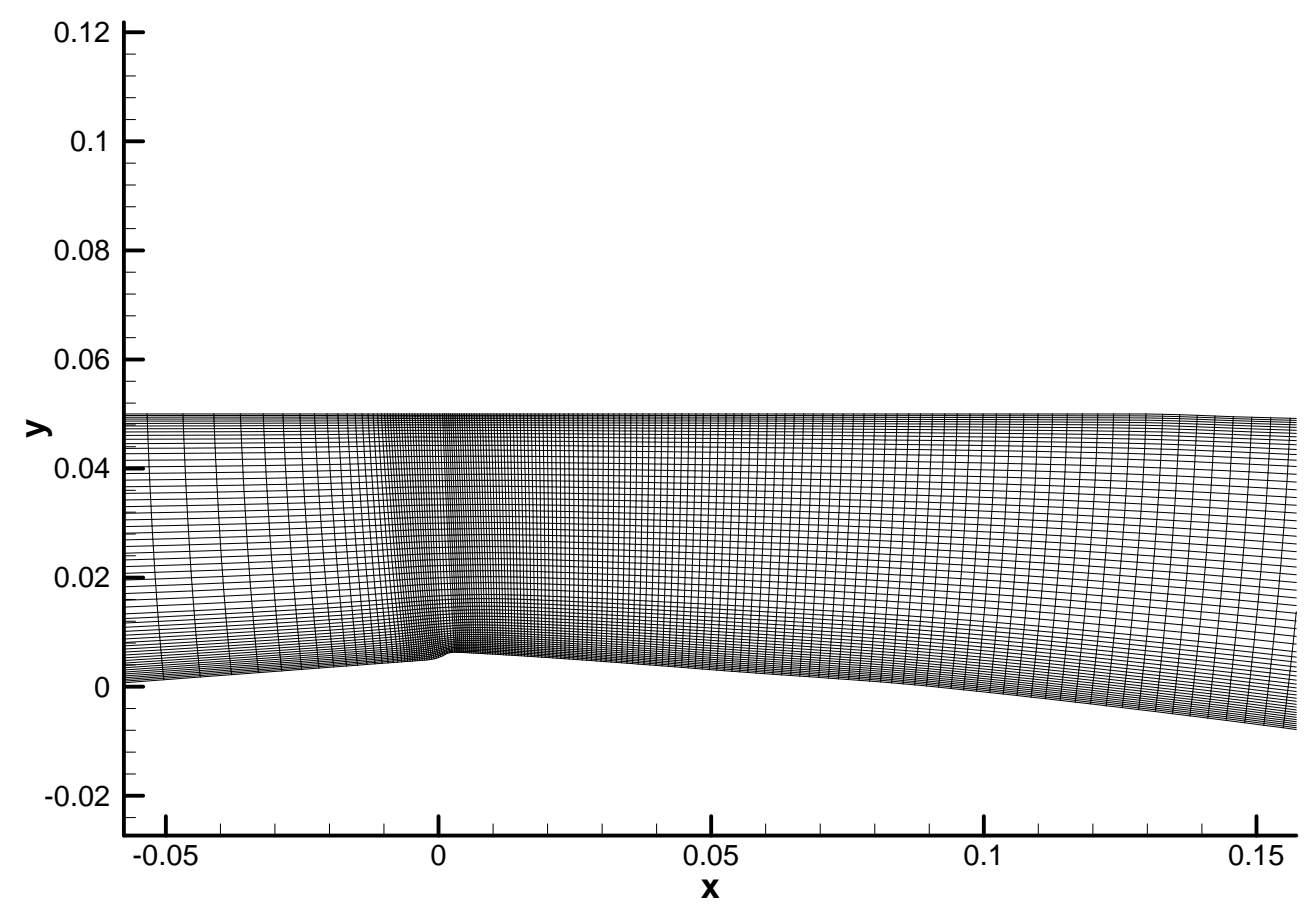

Fig. 4. Enlargement of the mesh near the throat, flat roof Venturi. 


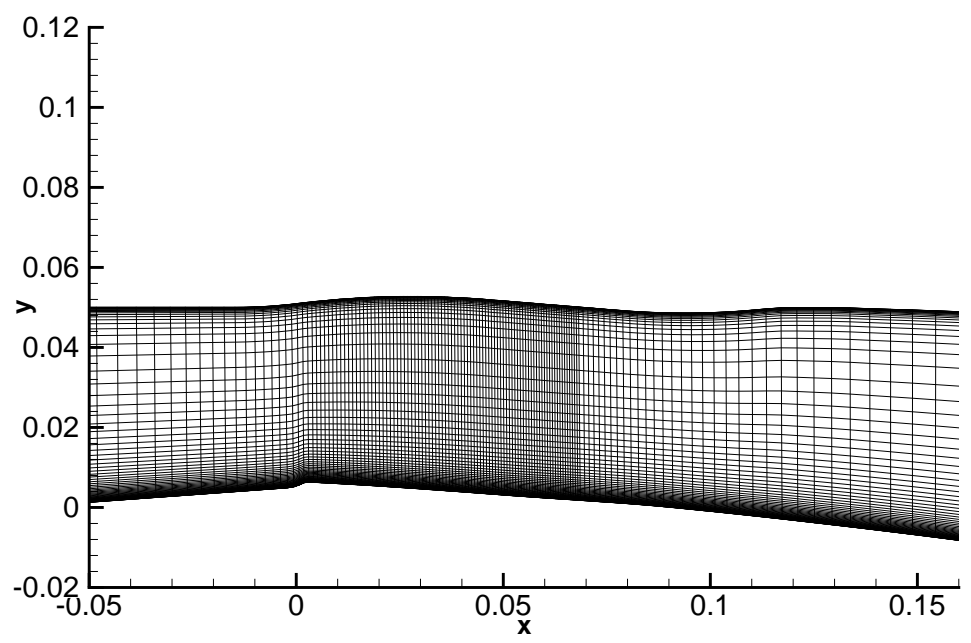

Fig. 5. Enlargement of the mesh near the throat, undulated roof Venturi. 

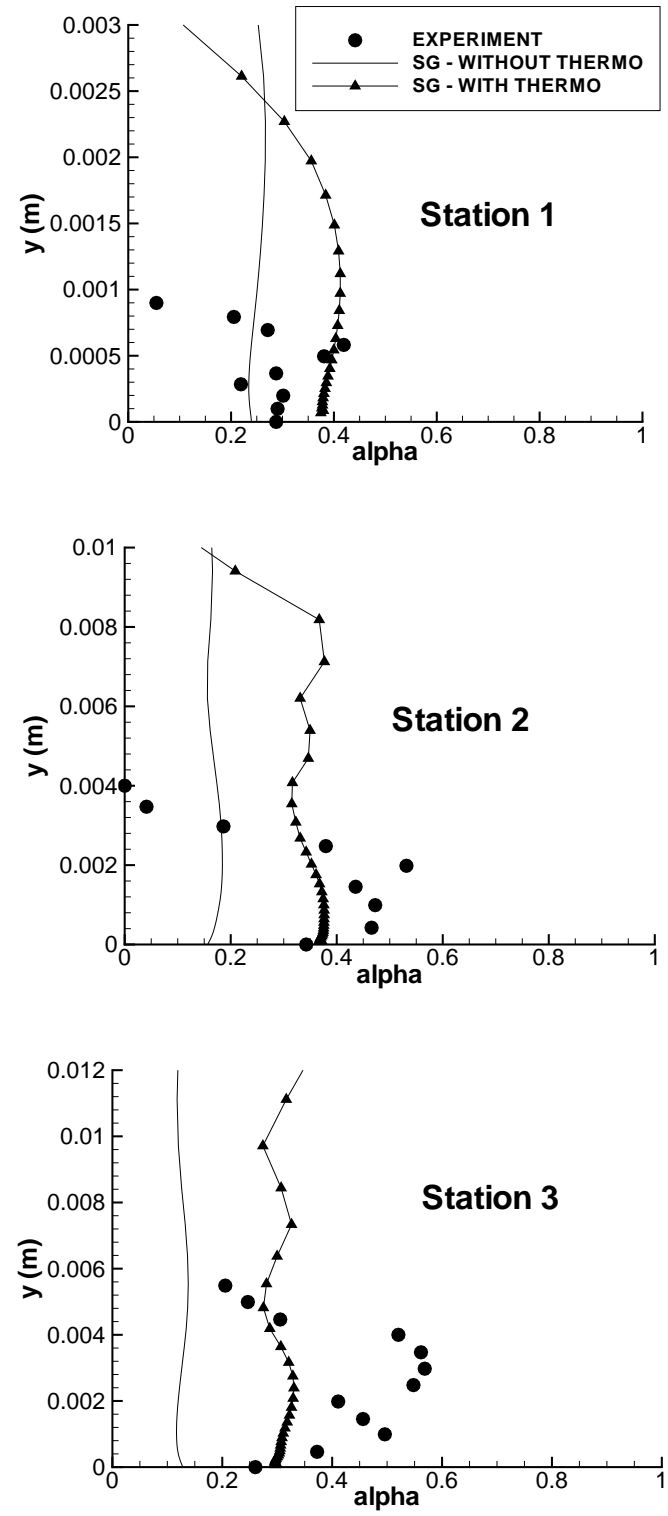

Fig. 6. Void ratio profiles for the stiffened gas EOS, flat roof computations. 


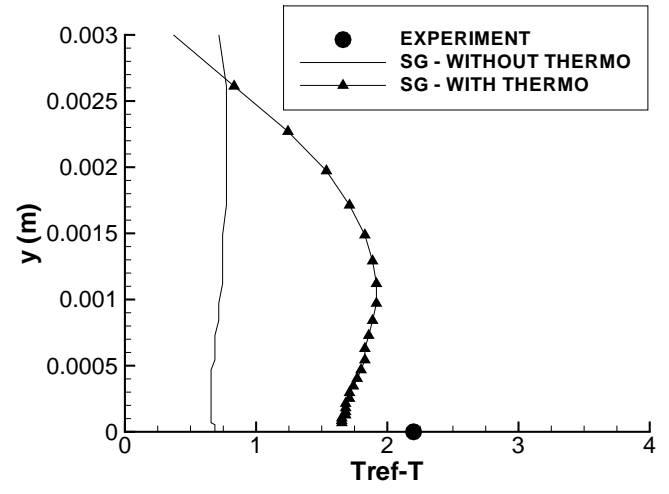

Fig. 7. Wall temperature depression at station 1, stiffened gas EOS, flat roof computations. 

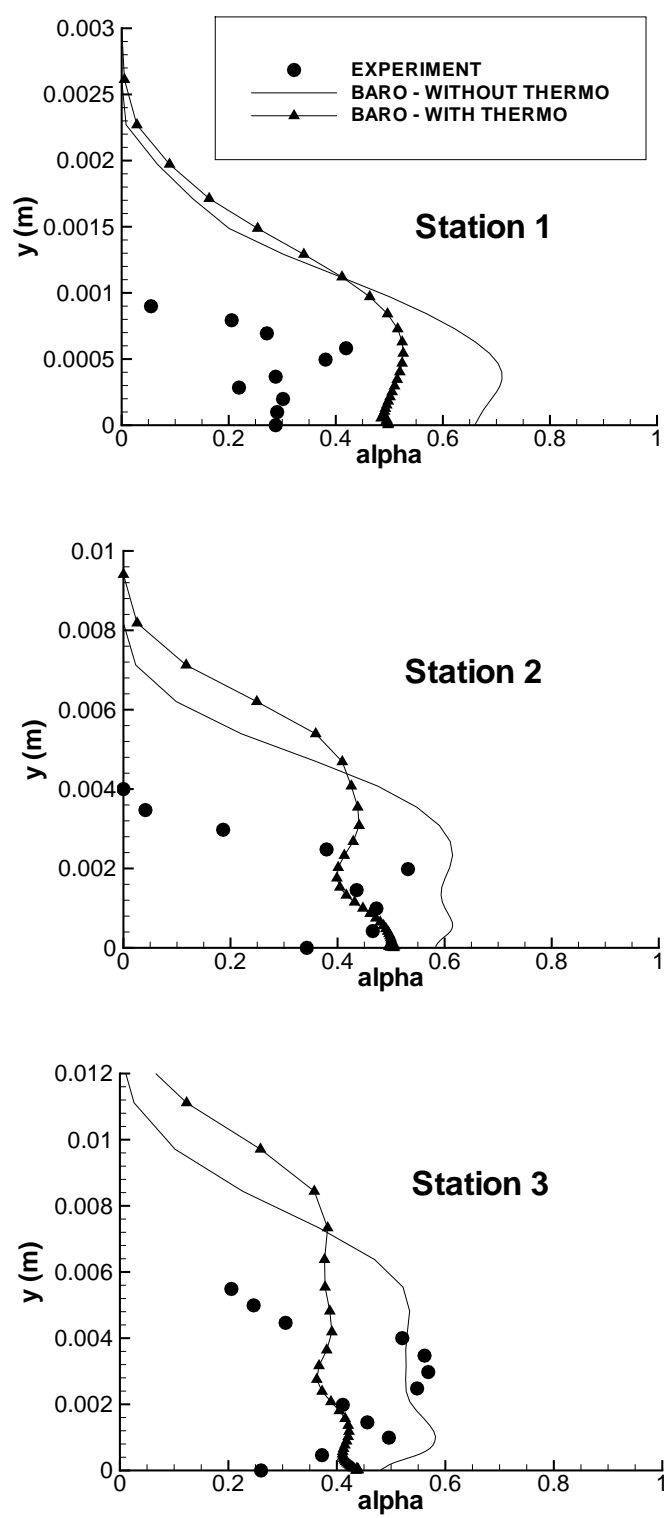

Fig. 8. Void ratio profiles, barotropic EOS with $c_{\text {baro }}=2.61 \mathrm{~m} / \mathrm{s}$, flat roof computations. 

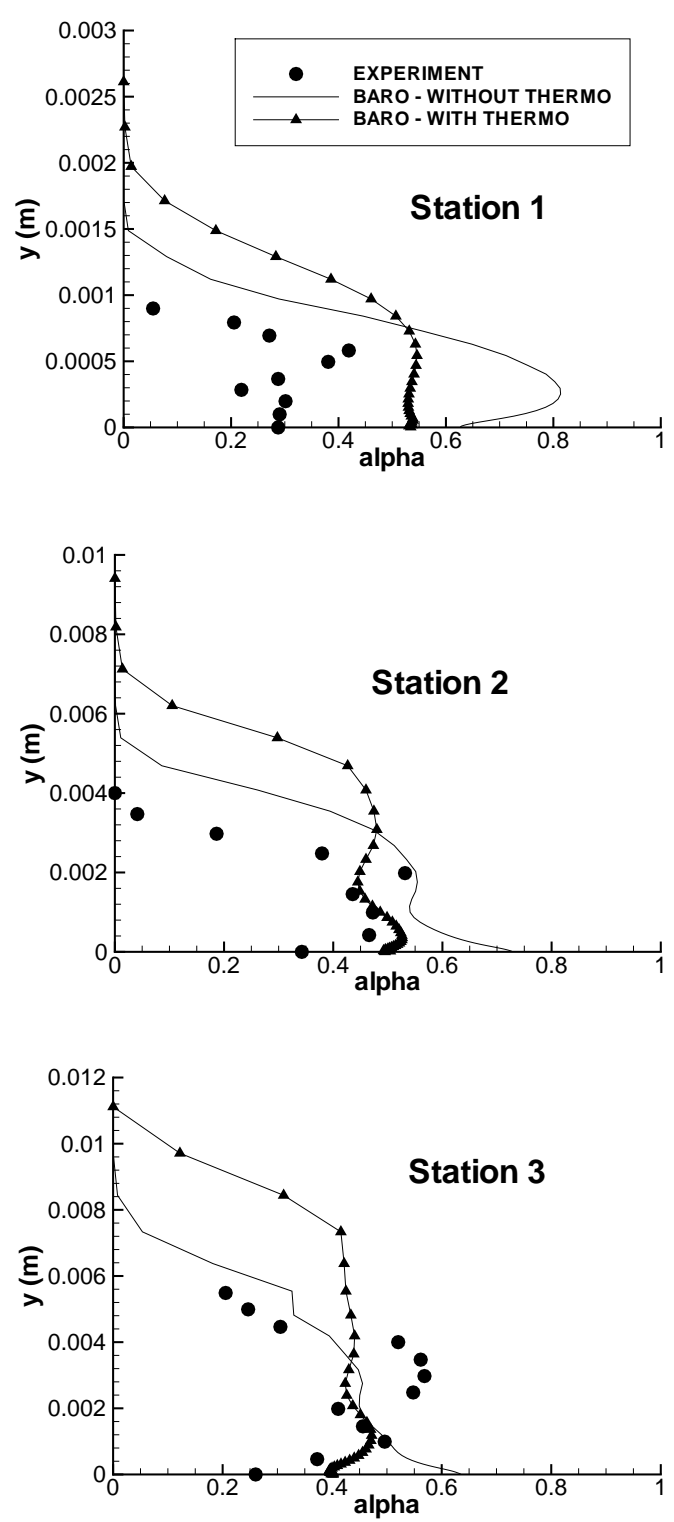

Fig. 9. Void ratio profiles, barotropic EOS with $c_{\text {baro }}=1.48 \mathrm{~m} / \mathrm{s}$, flat roof computations. 

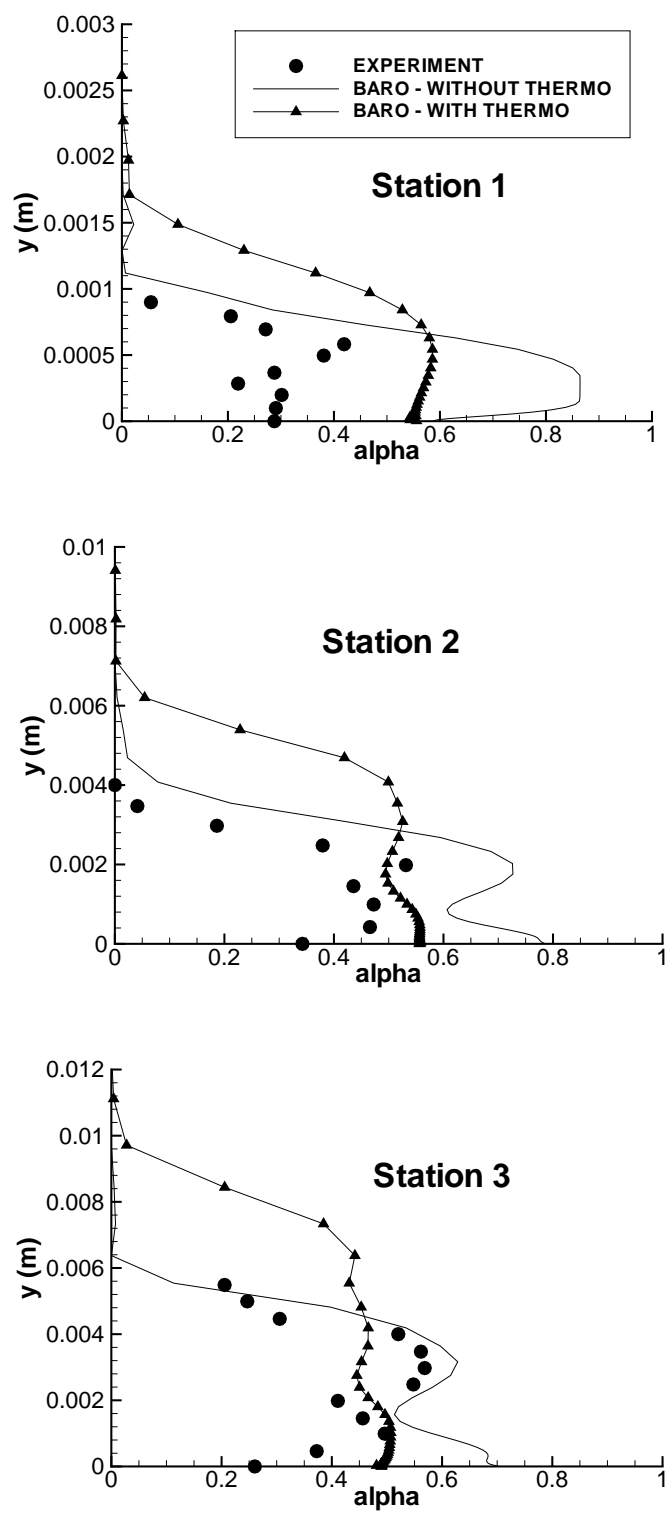

Fig. 10. Void ratio profiles, barotropic EOS with $c_{\text {baro }}=0.74 \mathrm{~m} / \mathrm{s}$, flat roof computations. 

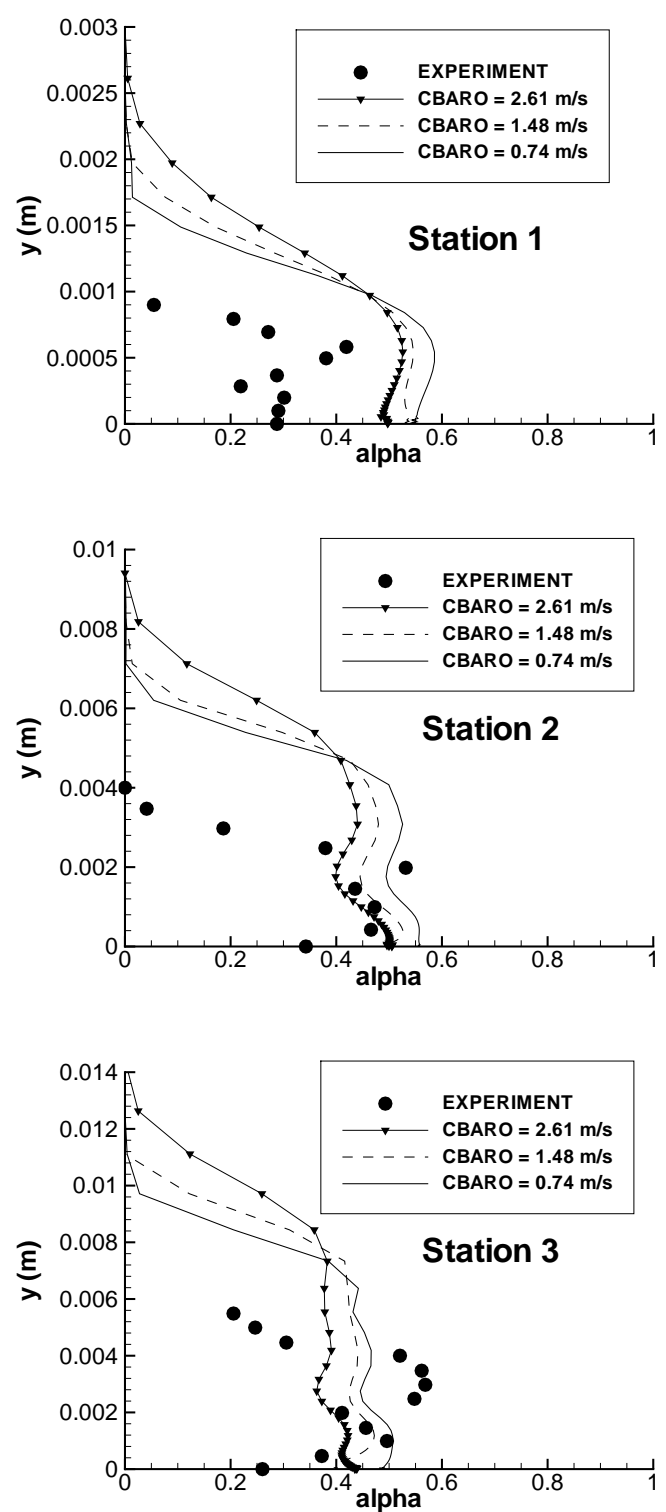

Fig. 11. Void ratio profiles, influence of $c_{\text {baro }}$, flat roof computations. 

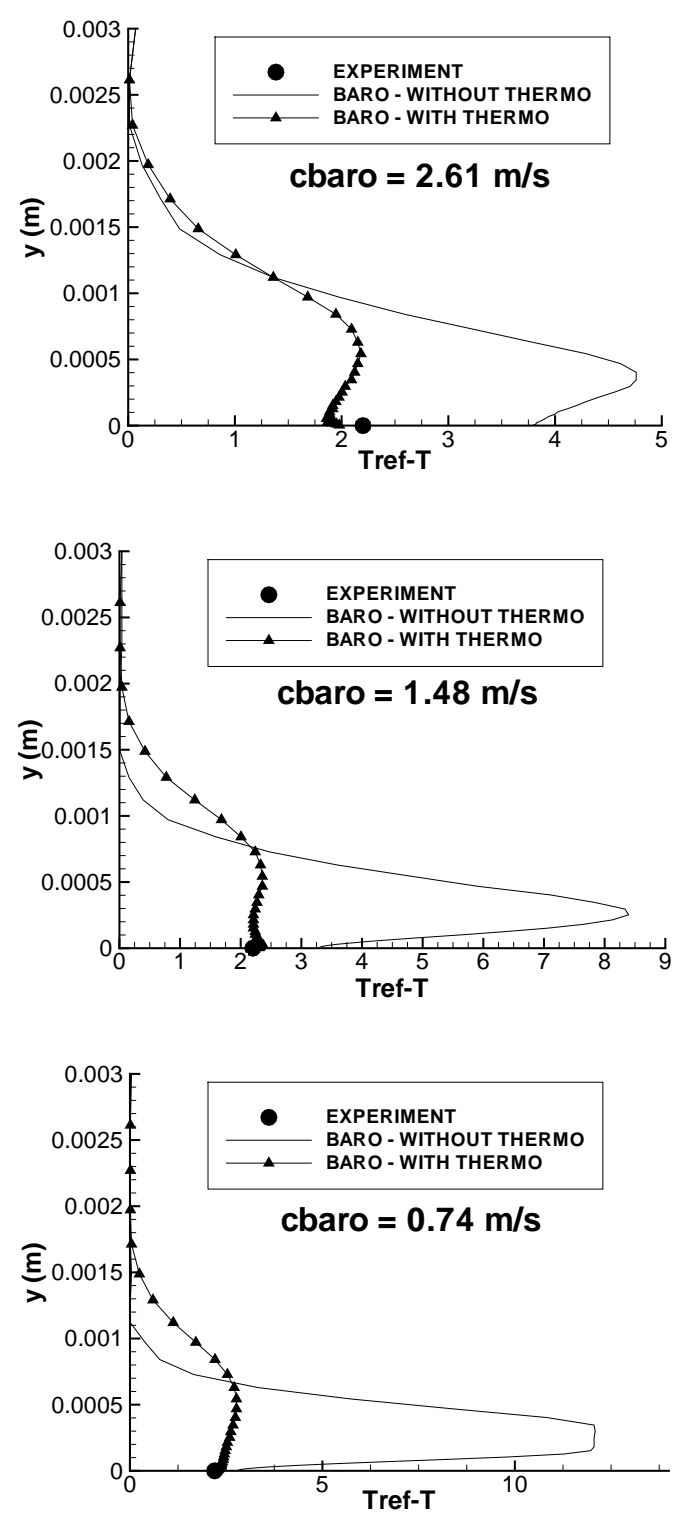

Fig. 12. Wall temperature depression at station 1, barotropic EOS, flat roof computations. 


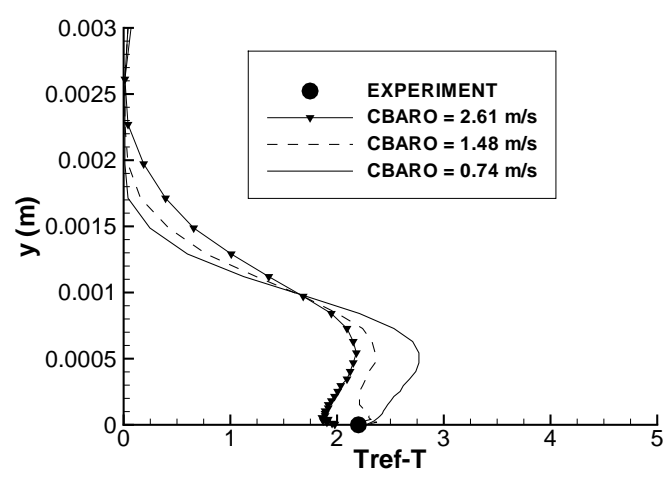

Fig. 13. Wall temperature depression at station 1 , influence of $c_{\text {baro }}$, flat roof computations. 

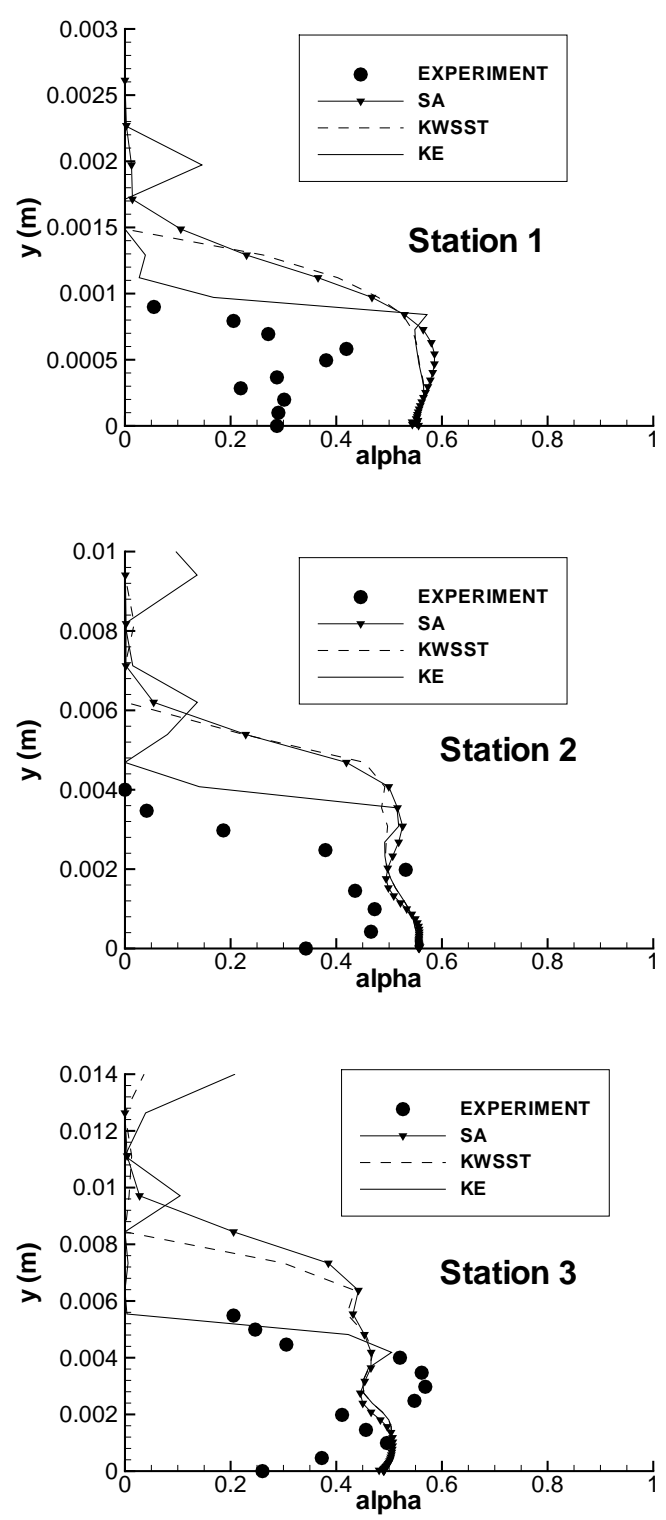

Fig. 14. Void ratio profiles, influence of the turbulence model, flat roof computations. 


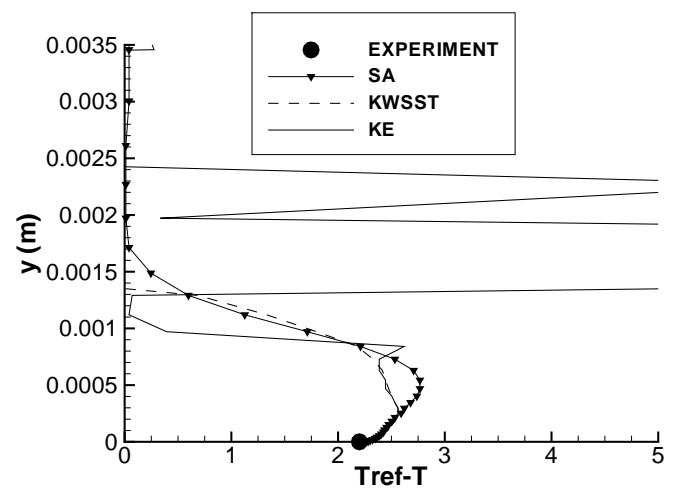

Fig. 15. Wall temperature depression at station 1, influence of the turbulence model, flat roof computations. 

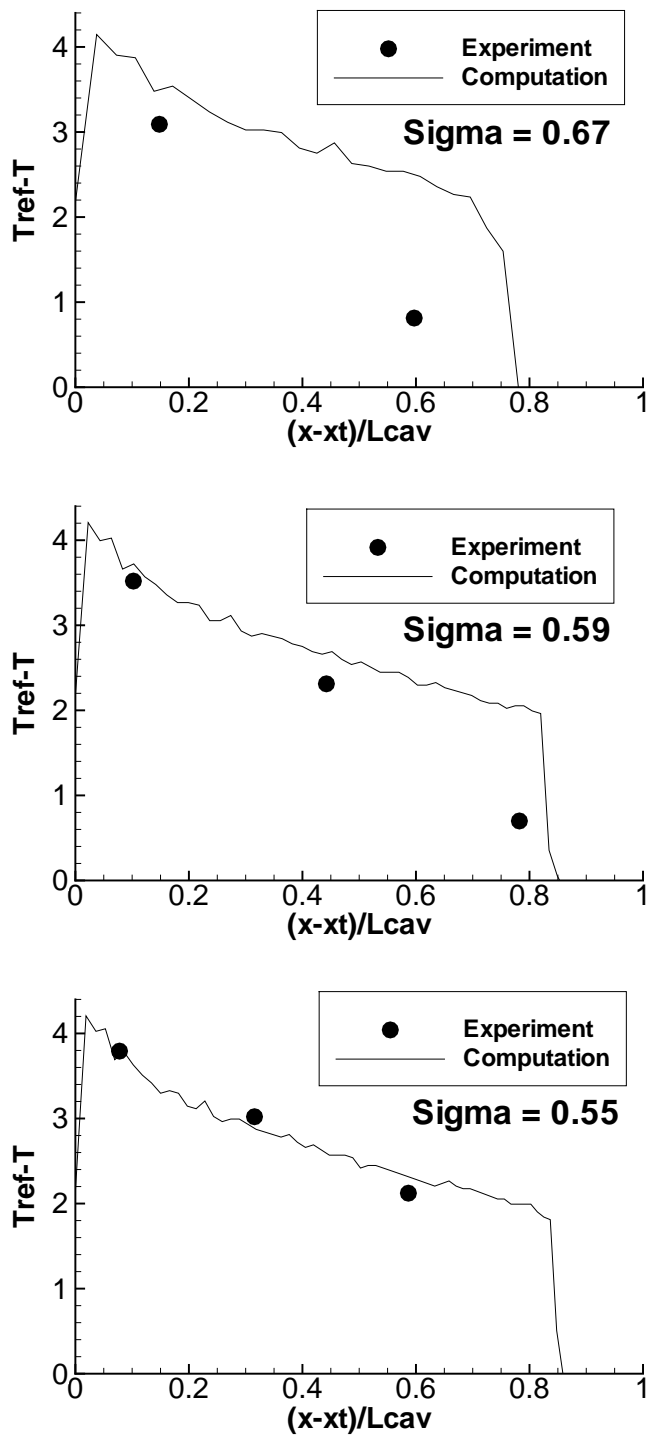

Fig. 16. Wall temperature depression, $T_{\text {ref }}=303 \mathrm{~K}$ and $V_{\text {inlet }}=22 \mathrm{~m} / \mathrm{s}$, $c_{\text {baro }}=0.74 \mathrm{~m} / \mathrm{s}$, undulated roof computations. 